\title{
The entanglement negativity in random spin chains
}

\author{
Paola Ruggiero, ${ }^{1}$ Vincenzo Alba, ${ }^{1}$ and Pasquale Calabrese ${ }^{1}$ \\ ${ }^{1}$ International School for Advanced Studies (SISSA), \\ Via Bonomea 265, 34136, Trieste, Italy, INFN, Sezione di Trieste
}

(Dated: May 4, 2016)

\begin{abstract}
We investigate the logarithmic negativity in strongly-disordered spin chains in the random-singlet phase. We focus on the spin- $1 / 2$ random Heisenberg chain and the random $X X$ chain. We find that for two arbitrary intervals the disorder-averaged negativity and the mutual information are proportional to the number of singlets shared between the two intervals. Using the strong-disorder renormalization group (SDRG), we prove that the negativity of two adjacent intervals grows logarithmically with the intervals length. In particular, the scaling behavior is the same as in conformal field theory, but with a different prefactor. For two disjoint intervals the negativity is given by a universal simple function of the cross ratio, reflecting scale invariance. As a function of the distance of the two intervals, the negativity decays algebraically in contrast with the exponential behavior in clean models. We confirm our predictions using a numerical implementation of the SDRG method. Finally, we also implement DMRG simulations for the negativity in open spin chains. The chains accessible in the presence of strong disorder are not sufficiently long to provide a reliable confirmation of the SDRG results.
\end{abstract}

\section{INTRODUCTION}

Entanglement measures are nowadays accepted as powerful tools to characterize quantum many-body systems ${ }^{1-4}$. Arguably, the most popular and useful one is the entanglement entropy. Given a pure state $|\psi\rangle$ and a bipartition into two parts $A$ and $B$, the entanglement entropy of part $A$ is defined as

$$
S_{A} \equiv-\operatorname{Tr} \rho_{A} \ln \rho_{A},
$$

where $\rho_{A} \equiv \operatorname{Tr}_{B}|\psi\rangle\langle\psi|$ is the reduced density matrix of $A$. For a pure state it is clear that $S_{A}=S_{B}$, reflecting the property that a good measure of entanglement is symmetric in $A$ and $B$.

If a system is in a mixed state, for instance at finite temperature, a useful measure of the correlation between $A$ and $B$ is the mutual information $\mathcal{I}_{A: B}$, which is defined as the symmetrized combination $\mathcal{I}_{A: B} \equiv S_{A}+S_{B}-S_{A \cup B}$. However, it is well known that the mutual information provides only an upper bound for the entanglement between $A$ and $B$, as it is sensitive to both classical and quantum correlations. A similar issue arises when quantifying the entanglement between disconnected regions in pure states. For instance, given the tripartition of a system as $B_{2} \cup A_{1} \cup B_{1} \cup A_{2} \cup B_{2}$ (as illustrated in Fig. 1 (a) for a spin chain), with $A \equiv A_{1} \cup A_{2}$ the region of interest, $\mathcal{I}_{A_{1}: A_{2}}$ is not a measure of the mutual entanglement between $A_{1}$ and $A_{2}$.

A computable measure of the mutual entanglement between two subsystems in a mixed state is provided by the so-called logarithmic negativity ${ }^{5-10}$

$$
\mathcal{E} \equiv \ln || \rho_{A}^{T_{2}} \|_{1}=\ln \operatorname{Tr}\left|\rho_{A}^{T_{2}}\right|
$$

Here $\rho_{A}^{T_{2}}$ is the partially transposed reduced density matrix with respect to $A_{2}$. This is defined as $\left\langle\varphi_{1} \varphi_{2}\left|\rho_{A}^{T_{2}}\right| \varphi_{1}^{\prime} \varphi_{2}^{\prime}\right\rangle \equiv\left\langle\varphi_{1} \varphi_{2}^{\prime}\left|\rho_{A}\right| \varphi_{1}^{\prime} \varphi_{2}\right\rangle$, with $\left\{\varphi_{1}\right\}$ and $\left\{\varphi_{2}\right\}$ being bases for $A_{1}$ and $A_{2}$, respectively.
Besides its interest in quantum information, recently the negativity became the focus of several interdisciplinary efforts to understand its role in many-body systems. For critical points described by a Conformal Field Theory (CFT) its scaling behavior has been derived analytically $^{11-13}$. This allowed to prove that, unlike the entanglement entropy, the negativity is scale invariant in gapless systems described by CFT, as it was already argued based on $\mathrm{DMRG}^{14-16}$ simulations ${ }^{17}$, on semi-analytic results for the harmonic chain ${ }^{18}$, and for mean-field exactly solvable spin models ${ }^{19}$. Furthermore, for disjoint intervals the logarithmic negativity contains, in principle, complete information about the intricate structure of the underlying $\mathrm{CFT}^{11}$ (as it does the entanglement entropy of two or more disjoint intervals ${ }^{21}$ ). Some of these results have been generalized to finite-temperature ${ }^{20}$, large central charge ${ }^{22}$, out of equilibrium situations ${ }^{23-26}$, and holographic $^{27}$ and massive quantum field theories ${ }^{28}$. In topologically ordered phases, i.e., characterized by a non-

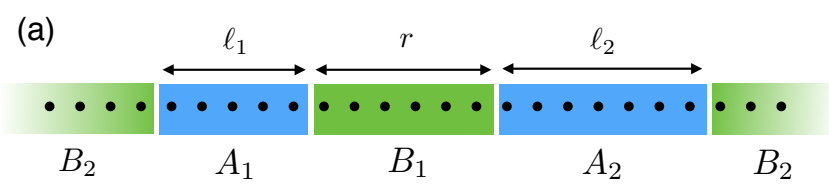

(b)

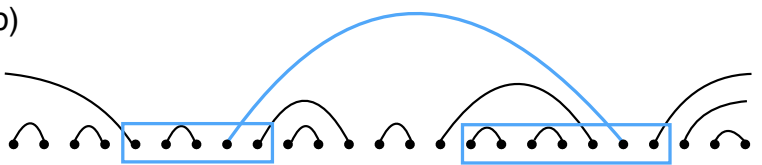

FIG. 1. (a): Partition of an infinite spin chain corresponding to two disjoint intervals. The region of interest is $A_{1} \cup A_{2}$. Two adjacent intervals correspond to $r=0$. (b): Cartoon of the random singlet phase. The lines connect pairs of spins forming $S U(2)$ singlets. The entanglement negativity $\mathcal{E}_{A_{1}: A_{2}}$ is proportional to the number of singlets $n_{A_{1}: A_{2}}$ shared between $A_{1}$ and $A_{2}$ (here $n_{A_{1}: A_{2}}=1$ ). 
zero topological entropy ${ }^{29,30}$, it has been shown that the negativity is sensitive only to the "quantum" contribution $^{31,32}$, while it is zero in classical topologically ordered states. Also, the negativity proved to be a useful tool to characterize Kondo-like behavior in spin chains ${ }^{33-35}$. Surprisingly, the exact treatment of the partial transposition in free fermion models is an arduous task, and no exact results are known yet for the negativity, despite some recent progresses for the calculations of the moments of the partial transpose $\mathrm{e}^{25,36-40}$. Oppositely, the negativity can be calculated analytically for free bosonic models ${ }^{41}$. for which few results are also available in higher dimensions $^{42,43}$. Furthermore, some interesting results on the negativity in Chern-Simons theories have been provided recently ${ }^{44}$. Finally, from the numerical perspective, effective methods to calculate the negativity are available in the context of Tree Tensor Networks ${ }^{13}$, while the moments of the partially transposed reduced density matrix can be calculated using classical ${ }^{45}$ and quantum ${ }^{46}$ Monte Carlo techniques. When not analytically possible, numerical extrapolations can be used to obtain the negativity from the replica limit of the moments ${ }^{47}$.

At the same time the study of the interplay between disorder and entanglement became a fruitful research $\operatorname{area}^{4,48}$. For instance, in Ref. 49 it has been shown that, for disordered spin chains exhibiting the random singlet (RS) phase, the scaling of the disorder averaged entanglement entropy is logarithmic with the subsystem size like in a CFT. This has been tested numerically in the random $X X$ chain $^{50}$ (which is exactly solvable for each realization of the disorder), and in the random $X X X$ chain $^{51}$ using DMRG. Furthermore, the moments of the reduced density matrix $\operatorname{Tr} \rho_{A}^{\alpha}$ have been also studied ${ }^{52}$, as well as the spectrum $^{53}$, and the entanglement in low-lying excited states $^{54}$. Other disordered spin models ${ }^{55-67}$ have been also considered, obtaining similar results for the scaling of the entanglement entropy. The non-equilibrium features of the entanglement in these random spin chains are also under intensive investigation ${ }^{51,68-75}$. The behavior of entanglement related quantities in classical disordered spin systems has been explored ${ }^{76}$.

In this paper we investigate the disorder averaged logarithmic negativity in RS phases in the framework of the strong-disorder renormalization group (SDRG) ${ }^{77}$. We focus on the spin- $1 / 2$ Heisenberg $(X X X)$ chain with random antiferromagnetic couplings, and on the random $X X$ chain. We consider both adjacent and disjoint intervals (see Fig. 1 (a)). We demonstrate that in a RS phase the negativity of two intervals is always proportional to the number of singlets shared between them. Surprisingly, due to the simple structure of the RS phase, this is also the case for the mutual information, which is given as $\mathcal{I}_{A_{1}: A_{2}}=2 \mathcal{E}_{A_{1}: A_{2}}$. More quantitatively, we find that for two adjacent intervals embedded in an infinite chain the disorder-averaged negativity scales as

$$
\mathcal{E}_{A_{1}: A_{2}}=\frac{\ln 2}{6} \ln \left(\frac{\ell_{1} \ell_{2}}{\ell_{1}+\ell_{2}}\right)+k,
$$

for large $\ell_{1}$ and $\ell_{2}$ (the lengths of the two intervals). Here $k$ is an additive constant. Interestingly, Eq. (3) has the same functional dependence on $\ell_{1,2}$ as in $\mathrm{CFT}^{11}$, but the prefactor of the logarithm is different. For two disjoint intervals (see Fig. 1 (a)), we obtain

$$
\mathcal{E}_{A_{1}: A_{2}}=\frac{\ln 2}{6} \ln \frac{\left(\ell_{1}+r\right)\left(\ell_{2}+r\right)}{r\left(\ell_{1}+\ell_{2}+r\right)}=-\frac{\ln 2}{6} \ln (1-x) .
$$

Here $\ell_{1}, \ell_{2}$ are the intervals length, and $r$ their distance. Eq. (4) is scale invariant and for this reason the r.h.s. has been written as a function of the cross ratio

$$
x=\frac{\ell_{1} \ell_{2}}{\left(\ell_{1}+r\right)\left(\ell_{1}+r\right)} .
$$

Again, this is similar to the CFT case, where $\mathcal{E}_{A_{1}: A_{2}}$ depends in a more intricate way on $x^{11,12}$. Interestingly, in the limit of two intervals far apart, from (4) one has the power-law decay $\mathcal{E} \propto r^{-2}$, in contrast with the CFT case, where this decay is exponential ${ }^{11}$. Both (3) and (4) turn out to be in perfect agreement with exact results obtained from a numerical implementation of the SDRG for the random $X X X$ chain.

Finally, by generalizing the method of Ref. 17, we discuss how to obtain the logarithmic negativity in DMRG simulations ${ }^{78}$ for arbitrary tripartitions of the spin chain. The computational cost of the algorithm is $\propto \chi_{\max }^{6}$, with $\chi_{\max }$ the maximal bond dimension of the matrix product state (MPS). As a byproduct of our analysis we numerically verify the CFT scaling of the negativity of two adjacent intervals in the clean $X X$ chain. In the presence of randomness it is numerically challenging to ensure the convergence of DMRG, introducing a systematic error in the numerical data. The latter is negligible only for small chains and weak disorder. The accessible chain sizes are not sufficient to provide reliable numerical evidence for (3) and (4).

The manuscript is organized as follows. In section II we introduce the $X X Z$ spin- $1 / 2$ chain with random antiferromagnetic couplings and summarize the strong disorder renormalization group (SDRG) method. In section III we provide the analytic expressions for the logarithmic negativity and the moments of the partially transposed reduced density matrix in RS phases. The scaling of the negativity is discussed in section IV for both adjacent and disjoint intervals. These results are verified in section $\mathrm{V}$ using a numerical implemtation of the SDRG method. Section VI focuses on the calculation of the logarithmic negativity in DMRG simulations. In section VII we present DMRG results for the negativity of two adjacent intervals in the random $X X$ chain. Finally, we conclude in section VIII.

\section{THE DISORDERED HEISENBERG SPIN CHAIN AND THE STRONG DISORDER RG}

The random antiferromagnetic spin- $\frac{1}{2} X X Z$ chain with open boundary conditions (OBC) is defined by the 
Hamiltonian

$$
\mathcal{H}=\sum_{i=1}^{L-1} J_{i}\left(S_{i}^{x} S_{i+1}^{x}+S_{i}^{y} S_{i+1}^{y}+\Delta S_{i}^{z} S_{i+1}^{z}\right),
$$

where $S_{i}^{x, y, z}$ are the spin components acting on site $i, L$ is the length of the chain, $\Delta$ the anisotropy parameter, and $\left\{J_{i}\right\}_{i=1}^{L-1}$ are uncorrelated positive random variables, drawn from a distribution $P(J)$. For periodic boundary conditions (PBC) one has an extra term in (6) connecting site $L$ with site 1 . We focus on $\Delta=1$ and $\Delta=0$, corresponding to the $X X X$ and the $X X$ random chain, respectively. For generic $\left\{J_{i}\right\}_{i=1}^{L-1}$, the latter can be treated analytically for each realization of the disorder, exploiting the mapping to free fermions (see Appendix A). Here we restrict ourselves to the family of distributions

$$
P_{\delta}(J) \equiv \delta^{-1} J^{-1+1 / \delta},
$$

with $J \in[0,1]$, and $0 \leq \delta<\infty$ a parameter tuning the disorder strength. For $\delta \rightarrow 0$ one recovers the clean, i.e., without disorder, $X X Z$ chain, whereas $\delta \rightarrow \infty$ corresponds to the infinite-randomness fixed point (IRFP, see section II). The latter describes the low-energy physics of (6), irrespective of the chosen distribution $P(J)$. For $\delta=1, P_{\delta}(J)$ becomes the flat distribution (box distribution) in the interval $[0,1]$.

We now briefly review the SDRG method for the $X X X$ chain. The main idea is to obtain a low-energy effective description of (6) by successively integrating out the strongest couplings, and renormalizing the remaining ones. Given an arbitrary coupling configuration $\left\{J_{i}\right\}_{i=1}^{L-1}$, one starts by identifying the strongest bond $J_{M} \equiv \max _{i}\left\{J_{i}\right\}$. The interaction between the two spins coupled by $J_{M}$ (that we denote as $\vec{S}_{l}$ and $\vec{S}_{r}$ ) is given by the Hamiltonian $\mathcal{H}_{0}$ as

$$
\mathcal{H}_{0}=J_{M} \vec{S}_{l} \cdot \vec{S}_{r}
$$

The ground state of $\mathcal{H}_{0}$ is the singlet state $|s\rangle$

$$
|s\rangle \equiv 2^{-1 / 2}\left(\left|\uparrow_{l} \downarrow_{r}\right\rangle-\left|\downarrow_{l} \uparrow_{r}\right\rangle\right) .
$$

The interaction between $\vec{S}_{l}$ and $\vec{S}_{r}$, and their neighboring spins (here denoted as $S_{l}^{\prime}$ and $S_{r}^{\prime}$, respectively) is described by the Hamiltonian $\mathcal{H}^{\prime}$ as

$$
\mathcal{H}^{\prime}=J_{l} \vec{S}_{l}^{\prime} \cdot \vec{S}_{l}+J_{r} \vec{S}_{r} \cdot{\overrightarrow{S^{\prime}}}_{r} .
$$

Since by definition $J_{l}, J_{r}<J_{M}$, one can treat $\mathcal{H}^{\prime}$ as a perturbation. Within second-order perturbation theory, this leads to the effective Hamiltonian $\mathcal{H}^{e f f}$ for ${\overrightarrow{S_{l}^{\prime}}}_{l}^{\prime}, \vec{S}_{r}^{\prime}$ as

$$
\begin{aligned}
\mathcal{H}^{e f f}=\left\langle s\left|\mathcal{H}_{0}+\mathcal{H}^{\prime}\right| s\right\rangle+\sum_{t} \frac{\left|\left\langle s\left|\mathcal{H}^{\prime}\right| t\right\rangle\right|^{2}}{E_{s}-E_{t}} & \\
& \equiv E_{0}+J^{\prime} \vec{S}_{l}^{\prime} \cdot \vec{S}_{r}^{\prime} .
\end{aligned}
$$

Here the sum is over the triplet states of two spins $|t\rangle=|\uparrow \uparrow\rangle,(|\uparrow \downarrow\rangle+|\downarrow \uparrow\rangle) / \sqrt{2},|\downarrow \downarrow\rangle$. The corresponding energies are $E_{t} \equiv\left\langle t\left|\mathcal{H}_{0}\right| t\right\rangle=1 / 4 J_{M}$, whereas one has $E_{s} \equiv\left\langle s\left|\mathcal{H}_{0}\right| s\right\rangle=-3 / 4 J_{M}$. In the last step in (11) we defined $E_{0}=-3\left(4 J_{M}+3 J_{l}^{2}+3 J_{r}^{2}\right) / 16$. The effective coupling $J^{\prime}$ between $\vec{S}_{l}^{\prime}$ and $\vec{S}_{r}^{\prime}$ reads

$$
J^{\prime}=\frac{J_{l} J_{r}}{2 J_{M}} .
$$

Note that (11) does not depend on $\vec{S}_{l}, \vec{S}_{r}$ anymore. Moreover, $\mathcal{H}^{e f f}$ is still of the Heisenberg form (6) with the renormalized coupling $J^{\prime}$. This process of decimating the spins connected by the strongest bond, renormalizing the remaining interactions, can be represented as

$$
\left(\cdots, J_{l}, J_{M}, J_{r}, \cdots\right)_{L} \rightarrow\left(\cdots, \frac{J_{l} J_{r}}{2 J_{M}}, \cdots\right)_{L-2},
$$

and it defines the so-called Dasgupta-Ma rule ${ }^{79}$. Crucially, since $J_{l}, J_{r}<J_{M}$, the repeated application of (13) reduces the energy scale of the model.

The low-energy properties of the model are asymptotically, i.e., after many iterations of (13), described by the so-called random singlet (RS) phase. This is illustrated in Fig. 1 (b). In the RS phase all the spins are paired (as stressed by the links in the figure) in a random fashion. Paired spins form singlet. From (13) it is clear that longer range singlets are generated at later steps of the SDRG.

The RS phase can be quantitatively characterized through the asymptotic distribution of the couplings $\left\{J_{i}\right\}$. The iteration of the Dasgupta-Ma rule (13) leads to a flow for $P(J)$. It is convenient to introduce at the given SDRG step $m$ the variables $\beta_{i}^{(m)}$ and $\Gamma^{(m)}$ as

$$
\beta_{i}^{(m)} \equiv \ln \frac{J_{M}^{(m)}}{J_{i}^{(m)}}, \quad \Gamma^{(m)} \equiv \ln \frac{J_{M}^{(0)}}{J_{M}^{(m)}} .
$$

Here $J_{M}^{(m)}$ is the maximum coupling at the step $m$. The physical interpretation is that $\Gamma^{(m)}$ quantifies the difference in energy scales between the initial step and step $m$, while the $\beta_{i}$ measure the broadness of the energy scales at a fixed step $m$. The equation describing the SDRG flow of $P(J)$ is given as ${ }^{80}$

$$
\begin{aligned}
& \frac{d P}{d \Gamma}=\frac{\partial P(\beta)}{\partial \beta} P(0) \times \\
& \quad \times \int_{0}^{\infty} d \beta_{1} \int_{0}^{\infty} d \beta_{2} \delta\left(\beta-\beta_{1}-\beta_{2}\right) P\left(\beta_{1}\right) P\left(\beta_{2}\right) .
\end{aligned}
$$

It can be shown that (15) has a unique solution $P^{*}(\beta)$ given by

$$
P^{*}(\beta)=\frac{1}{\Gamma} \exp \left(-\frac{\beta}{\Gamma}\right)
$$

Here $P^{*}(\beta)$ represents the fixed point of the SDRG flow. This fixed point is known as infinite randomness fixed point (IRFP) ${ }^{80}$, to emphasize that the broadness of the 
distribution increases during the flow. This is reflected in $P^{*}(\beta)$ being flat, which corresponds to $P^{*}(J)$ being peaked at $J=0$ (cf. (14)). Note that this justifies the perturbative treatment ${ }^{81}$ of $\mathcal{H}^{\prime}$ in (11). Remarkably, (16) does not depend on the initial distribution which is a manifestation of its universality.

Finally, we remind the reader that for the $X X$ chain $(\Delta=0$ in (6)) the Dasgupta-Ma rule (13) has to be modified as

$$
\left(\cdots, J_{l}, J_{M}, J_{r}, \cdots\right)_{L} \rightarrow\left(\cdots, \frac{J_{l} J_{r}}{J_{M}}, \cdots\right)_{L-2} .
$$

Consequently, the flow equation (15), and the fixed point distribution (16) remain the same as in the XXX case.

\section{LOGARITHMIC NEGATIVITY IN RANDOM SINGLET PHASES}

For a generic realization of the disorder, assuming that the chain is in a RS phase, the logarithmic negativity between two subsystems of the chain can be calculated analytically. In this section we show that it is proportional to the number of singlets shared between the two intervals. Let us consider a partition of the chain as in Fig. 1 with $A_{1} \cup A_{2}$ the two intervals of interest and $B_{1} \cup B_{2}$ their complement. For later convenience let us define

$$
A=A_{1} \cup A_{2}, \quad B=B_{1} \cup B_{2} .
$$

Given any two blocks $X, Y$ in the chain, we denote as $n_{X: Y}$ the number of singlets shared between them.

Before considering the entire RS phase, it is instructive to write down the density matrix and its partial transpose for an isolated singlet (more generic situations have been also considered in the literature ${ }^{82}$ ). The density matrix $\rho_{2 S}$ of two spins forming a singlet is

$$
\rho_{2 S}=\frac{1}{2}\left(\begin{array}{cccc}
0 & 0 & 0 & 0 \\
0 & 1 & -1 & 0 \\
0 & -1 & 1 & 0 \\
0 & 0 & 0 & 0
\end{array}\right)
$$

in the basis $|\uparrow \uparrow\rangle,|\uparrow \downarrow\rangle,|\downarrow \uparrow\rangle$, and $|\downarrow \downarrow\rangle$. The reduced density matrix $\rho_{S}$ for one of the spins is

$$
\rho_{S}=\frac{1}{2}\left(\begin{array}{ll}
1 & 0 \\
0 & 1
\end{array}\right)
$$

In order to calculate the negativity, we need the partial transpose $\rho_{2 S}^{T_{2}}$ that is

$$
\rho_{2 S}^{T_{2}}=\frac{1}{2}\left(\begin{array}{cccc}
0 & 0 & 0 & -1 \\
0 & 1 & 0 & 0 \\
0 & 0 & 1 & 0 \\
-1 & 0 & 0 & 0
\end{array}\right)
$$

with eigenvalues $\{-1 / 2,1 / 2,1 / 2,1 / 2\}$.
The density matrix $\rho_{R S P}$ for a chain in a random singlet phase is the tensor product of the density matrices of its constituent singlets. Thus, for the partition in Fig. 1, one can write

$$
\rho_{R S P}=\bigotimes_{i=1}^{n_{A: A}} \rho_{2 S} \bigotimes_{i=1}^{n_{B: B}} \rho_{2 S} \bigotimes_{i=1}^{n_{A: B}} \rho_{2 S}
$$

The reduced density matrix $\rho_{A}$ is obtained from (22) as

$$
\rho_{A}=\bigotimes_{i=1}^{n_{A: A}} \rho_{2 S} \bigotimes_{i=1}^{n_{A: B}} \rho_{S}
$$

and its partial transpose

$$
\rho_{A}^{T_{2}}=\bigotimes_{i=1}^{n_{A: A}} \rho_{2 S}^{T_{2}} \bigotimes_{i=1}^{n_{A: B}} \rho_{S}^{T_{2}}
$$

To compute the negativity $\mathcal{E}_{A_{1}: A_{2}}$ one can exploit the additivity of the negativity on tensor products, i.e., that $\mathcal{E}\left(\otimes_{i} \rho_{i}\right)=\sum_{i} \mathcal{E}\left(\rho_{i}\right)$. From (24) one then has

$$
\begin{aligned}
& \mathcal{E}_{A_{1}: A_{2}}=n_{A_{1}: A_{2}} \ln \operatorname{Tr}\left|\rho_{2 S}^{T_{2}}\left(A_{1} \cup A_{2}\right)\right| \\
& +\sum_{i=1,2}\left[n_{A_{i}: A_{i}} \ln \operatorname{Tr}\left|\rho_{2 S}^{T_{2}}\left(A_{i}\right)\right|+n_{A_{i}: B} \ln \operatorname{Tr}\left|\rho_{S}^{T_{2}}\left(A_{i}\right)\right|\right]
\end{aligned}
$$

Interestingly, the terms in the square brackets vanish because for any $A_{i}, \rho_{2 S}^{T_{2}}\left(A_{i}\right)=\rho_{2 S}\left(A_{i}\right)$ and $\rho_{S}^{T_{2}}\left(A_{i}\right)=$ $\rho_{S}\left(A_{i}\right)$. As a consequence, $\mathcal{E}_{A_{1}: A_{2}}$ depends only on the number of singlets $n_{A_{1}: A_{2}}$ shared between $A_{1}$ and $A_{2}$. Physically, this could have been expected because the negativity is a measure of the mutual entanglement between $A_{1}$ and $A_{2}$. In conclusion, we have

$$
\mathcal{E}_{A_{1}: A_{2}}=n_{A_{1}: A_{2}} \ln \operatorname{Tr}\left|\rho_{2 S}^{T_{2}}\left(A_{1} \cup A_{2}\right)\right|,
$$

and using the explicit form of $\rho_{2 S}^{T_{2}}(21)$

$$
\mathcal{E}_{A_{1}: A_{2}}=n_{A_{1}: A_{2}} \ln 2 .
$$

For a bipartition, i.e., for $A_{2} \equiv \overline{A_{1}}$, with $\bar{A}_{1}$ the complement of $A_{1}$, Eq. (26) is equal to the entanglement entropy of $A_{1}$ (see Ref. 49). This is just a consequence of the fact that in the RS phase the Rényi entropies $S_{\alpha}$ with varying $\alpha$ are all equal ${ }^{52}$, while in the generic case the negativity for a bipartition is always equal to $S_{1 / 2}{ }^{9,12}$. This is different from the clean bipartite system (which is conformal invariant) in which the Rényi entropies depend non trivially on the index $\alpha$ in a well-known fashion ${ }^{83,84}$. Finally, it is interesting to compare (26) with the mutual information between two intervals $\mathcal{I}_{A_{1}: A_{2}}=S_{A_{1}}+$ $S_{A_{2}}-S_{A_{1} \cup A_{2}}$ which can be readily obtained using $S_{X}=$ $n_{X: \bar{X}} \ln 2$. A straightforward calculation yields

$\mathcal{I}_{A_{1}: A_{2}}=\left(n_{A_{1}: \bar{A}_{1}}+n_{A_{2}: \bar{A}_{2}}-n_{A_{1} \cup A_{2}: B}\right) \ln 2=2 n_{A_{1}: A_{2}} \ln 2$.

Interestingly, (27) coincides, apart from a factor 2, with $\mathcal{E}_{A_{1}: A_{2}}$ in (26). 


\section{A. The moments of the partially transposed reduced density matrix}

It is instructive to consider the moments $M_{\alpha}^{T_{2}}$ of the partially transposed density matrix

$$
M_{\alpha}^{T_{2}} \equiv \operatorname{Tr}\left(\rho_{A}^{T_{2}}\right)^{\alpha}
$$

The logarithmic negativity can be obtained from (28) via the analytic continuation $\alpha \rightarrow 1$ restricted to the even $\alpha$, as it is routinely done in CFT calculations ${ }^{11,12}$. Although $M_{\alpha}^{T_{2}}$ are not entanglement measures, they encode universal information about critical systems. In particular, the moments $M_{\alpha}^{T_{2}}$ contain more information than the entanglement negativity, and, at least in principle, they could be used to characterize the entire spectrum of $\rho_{A}^{T 2}$ on the same lines as it has been done for reduced density matrix $^{85}$.

The computation of the moments starts by rewriting (23) as

$$
\rho_{A}=\left\{\prod_{k} \bigotimes_{i=1}^{n_{A_{k}: A_{k}}} \rho_{2 S}\right\} \bigotimes_{i=1}^{n_{A_{1}: A_{2}}} \rho_{2 S}\left\{\prod_{k} \bigotimes_{i=1}^{n_{A_{k}: B}} \rho_{S}\right\} .
$$

Using that the partial transposition acts trivially on the terms in the curly brackets, one obtains

$$
\rho_{A}^{T_{2}}=\left\{\prod_{k} \bigotimes_{i=1}^{n_{A_{k}: A_{k}}} \rho_{2 S}\right\} \bigotimes_{i=1}^{n_{A_{1}: A_{2}}} \rho_{2 S}^{T_{2}}\left\{\prod_{k} \bigotimes_{i=1}^{n_{A_{k}: B}} \rho_{S}\right\}
$$

The only two non-zero eigenvalues of $\rho_{A}^{T_{2}}$ are

$$
\lambda_{ \pm}= \pm 2^{-n_{A: B}-n_{A_{1}: A_{2}}}
$$

The corresponding degeneracies $d_{ \pm}$are

$$
\begin{aligned}
& d_{-}=\left(2^{n_{A_{1}: A_{2}}}-1\right) 2^{n_{A_{1}: A_{2}}-1}, \\
& d_{+}=2^{n_{A: B}+2 n_{A_{1}: A_{2}}}-d_{-} .
\end{aligned}
$$

The moments $M_{\alpha}^{T_{2}}$ can be readily written down as

$$
M_{\alpha}^{T_{2}}=2^{\left(n_{A: B}+n_{A_{1}: A_{2}}\right)(1-\alpha)} \begin{cases}2^{n_{A_{1}: A_{2}}} & \alpha \text { even } \\ 1 & \alpha \text { odd }\end{cases}
$$

In Eq. (34) it is evident that for generic $\alpha, M_{\alpha}^{T_{2}}$ cannot be measures of the entanglement between $A_{1}$ and $A_{2}$, since they depend on the number of singlets shared with $B$. By analytically continuing Eq. (34) from the even sequence we recover the negativity (26), i.e., denoting with $n_{e}$ the even integers, we have

$$
\mathcal{E}=\lim _{n_{e} \rightarrow 1} \ln M_{n_{e}}^{T_{2}},
$$

while the replica limit of the odd sequence gives the normalization $\operatorname{Tr} \rho_{A}^{T_{2}}=1^{11,12}$.

\section{SCALING OF THE NEGATIVITY IN THE RANDOM SINGLET PHASE}

In this section we derive the scaling properties of the logarithmic negativity between two intervals $A_{1}$ and $A_{2}$ in the random singlet phase. From the previous section, it is obvious that the only needed ingredient is the scaling of the average number of singlets $\left\langle n_{A_{1}: A_{2}}\right\rangle$ shared between $A_{1}$ and $A_{2}$ (cf. (26)). Here $\langle\cdot\rangle$ denotes the disorder average. Remarkably, $\left\langle n_{A_{1}: A_{2}}\right\rangle$ can be obtained from the result for a single interval ${ }^{49}$. Given an interval $X$ of length $\ell_{X}$ embedded in the infinite line, the average number of shared singlets $\left\langle n_{X: \bar{X}}\right\rangle$ scales for large $\ell_{X}$ as ${ }^{49}$

$$
\left\langle n_{X: \bar{X}}\right\rangle=\frac{1}{3} \ln \ell_{X}+k,
$$

where $k$ a non-universal constant.

In order to derive $\left\langle n_{A_{1}: A_{2}}\right\rangle$, let us consider a generic multipartition of the chain into $2 k$ blocks as $\cup_{Y \in \Omega_{0}} Y$, with $\Omega_{0}=\left\{A_{1}, B_{1}, \cdots A_{k}, B_{k}\right\}$ (the case with $k=2$ is in Fig. 1 (a)). It is convenient to define the set $\Omega$ of all possible compact subintervals of the chain. For instance, for $\Omega_{0}=\left\{A_{1}, B_{1}, A_{2}, B_{2}\right\}$ one has $\Omega=\left\{A_{1}, B_{1}, A_{2}, B_{2}, A_{1} \cup\right.$ $\left.B_{1}, B_{1} \cup A_{2}\right\}$. For each $X \in \Omega$, one can decompose the number of singlets $n_{X: \bar{X}}$ as the sum of all the singlets emerging from an arbitrary block $Y \in X$ and ending in an arbitrary block $Z \in \bar{X}$, i.e. in formula

$$
n_{X: \bar{X}}=\sum_{Y, Z \in \Omega_{0}} n_{(X \cap Y):(\bar{X} \cap Z)} .
$$

After taking the disorder average, (37) gives

$$
\frac{1}{3} \ln \ell_{X}+k=\sum_{Y, Z \in \Omega_{0}}\left\langle n_{(X \cap Y):(\bar{X} \cap Z)}\right\rangle,
$$

where we used that (36) is valid for any $X \in \Omega$. One can obtain $\left\langle n_{X: Y}\right\rangle$ for any pair $X, Y \in \Omega_{0}$ by solving the linear system of equations in (38) generated by considering all $X \in \Omega$, as we are going to show in the following for two adjacent and disjoint intervals.

\section{A. Two adjacent intervals}

For two adjacent inverals we have $\Omega_{0}=\Omega=$ $\left\{A_{1}, A_{2}, B_{2}\right\}$, and so the system of equations (38) becomes

$$
\left\{\begin{array}{l}
\left\langle n_{A_{1}: A_{2}}\right\rangle+\left\langle n_{A_{1}: B_{2}}\right\rangle=\frac{1}{3} \ln \left(\ell_{1}\right), \\
\left\langle n_{A_{2}: A_{1}}\right\rangle+\left\langle n_{A_{2}: B_{2}}\right\rangle=\frac{1}{3} \ln \left(\ell_{2}\right), \\
\left\langle n_{B_{2}: A_{1}}\right\rangle+\left\langle n_{B_{2}: A_{2}}\right\rangle=\frac{1}{3} \ln \left(\ell_{1}+\ell_{2}\right),
\end{array}\right.
$$

and the solution for $\left\langle n_{A_{1}: A_{2}}\right\rangle$ is

$$
\left\langle n_{A_{1}: A_{2}}\right\rangle=\frac{1}{6} \ln \left(\frac{\ell_{1} \ell_{2}}{\ell_{1}+\ell_{2}}\right) .
$$


Consequently [from (40) and (26)], one recovers the scaling of the logarithmic negativity in (3):

$$
\mathcal{E}_{A_{1}: A_{2}}=\frac{\ln 2}{6} \ln \left(\frac{\ell_{1} \ell_{2}}{\ell_{1}+\ell_{2}}\right)+k,
$$

\section{B. Two disjoint intervals}

Let us consider the case of two disjoint intervals (see Fig. 1 (a)) of lengths $\ell_{1}, \ell_{2}$, and at distance $r$, for which $\Omega=\left\{A_{1}, A_{2}, B_{1}, B_{2}, A_{1} \cup B_{1}, B_{1} \cup A_{2}\right\}$. From (38) one obtains a linear system of six equations with solution for $\left\langle n_{A_{1}: A_{2}}\right\rangle$ given by

$$
\left\langle n_{A_{1}: A_{2}}\right\rangle=\frac{1}{6} \ln \frac{\left(r+\ell_{1}\right)\left(r+\ell_{2}\right)}{r\left(\ell_{1}+\ell_{2}+r\right)} .
$$

Interestingly, (42) does not depend on the non-universal additive constant $k$ indicating that the logarithmic negativity between two disjoint intervals is a universal scale invariant quantity, as in the clean case. In particular, from (42), $\mathcal{E}_{A_{1}: A_{2}}$ can be rewritten as a function only of the cross ratio $x$ as in (4).

An intriguing consequence of (42) is that the entanglement between two disjoint intervals decays as $\propto r^{-2}$, i.e., with a power law of their mutual distance, in stark contrast with the CFT case where this decays is exponential $^{11}$. The result for adjacent intervals (40) is recovered from (42) by taking $r$ of the order of the lattice spacing (fixed to 1 in all above formulas) and then considering $\ell_{1,2}$ much larger than that.

\section{NUMERICAL SDRG}

In this section we present numerical evidence confirming the analytical results for the average number of singlets between two arbitrary intervals (adjacent and disjoint) and consequently for the negativity and mutual information between them. In order to do so, we numerical implement the SDRG for finite-size spin chains which works according to the following the steps: a) for a given disorder realization, we apply the Dasgupta-Ma rule (13), i.e., we pair the spins interacting with the strongest bond to form a $S U(2)$ singlet; b) the two spins are then decimated, and the remaining couplings renormalized according to $(12)$; c) this SDRG step is iterated until all the spins are paired in singlets. At every RG step we monitor the distribution of the renormalized couplings, as well as the spin configurations. In this section we only consider a flat probability distribution of the coupling $J$ between 0 and 1 .

\section{A. SDRG flow of the renormalized couplings}

As a preliminary check of the numerical SDRG, we study the flow of the couplings $J_{i}$ in (6) as a function of the

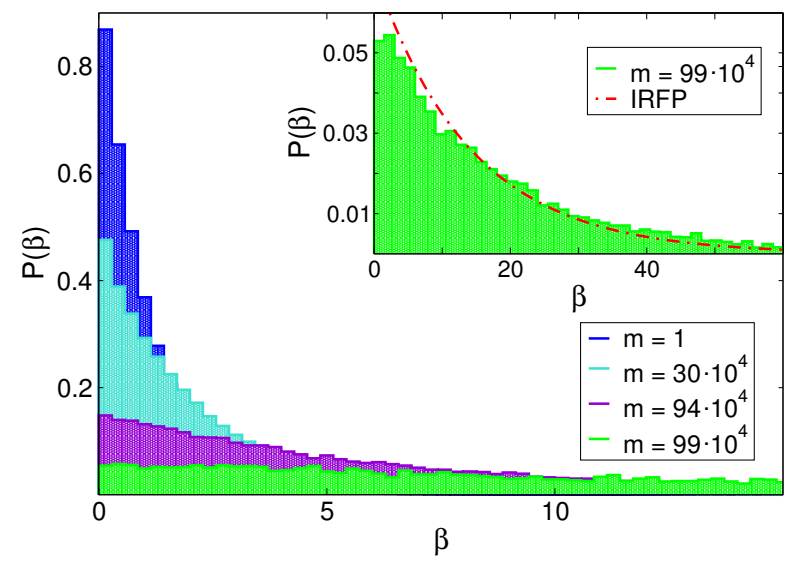

FIG. 2. Numerical strong disorder renormalization group: flow of the couplings probability distribution $P\left(\beta^{(m)}\right)$ as a function of the SDRG step $m$. Different histograms correspond to different $m$. Results are for the Heisenberg spin chain with $L=10^{6}$ sites. The width of $P\left(\beta_{i}^{(m)}\right)$ increases upon increasing $m$. Inset: $P\left(\beta^{(m)}\right)$ for $m=99 \cdot 10^{4}$, compared with the infinite-randomness fixed point (IRFP) result $P^{*}(\beta)=e^{-\beta / \Gamma} / \Gamma,\left(\right.$ dash-dotted line) with $\Gamma^{(m)}$ given in (14).

SDRG step $m$. The results are reported in Fig. 2, where we plot the probability distribution $P\left(\beta_{i}^{(m)}\right)$, with $\beta_{i}^{(m)} \equiv$ $\ln \left(J_{M}^{(m)} / J_{i}^{(m)}\right)$ (cf. (14)), and $J_{M}^{(m)}$ the maximum coupling at step $m$. The data are for a finite chain with $L=10^{6}$. The initial values of the couplings are drawn from the box distribution $[0,1]$.

Fig. 2 demonstrates the broadening of the couplings distribution upon increasing the SDRG step $m$, confirming that the SDRG procedure is asymptotically exact at large scale $^{81}$. The convergence to the universal IRFP distribution $P^{*}(\beta)$ (16) is verified in the inset of Fig. 2.

\section{B. Single-interval entanglement entropy}

Another important check of the validity of the numerical SDRG is provided by the scaling of the single block entanglement entropy. We consider the random $X X X$ chain with both open and periodic boundary conditions. For OBC we expect different scalings depending on whether the block $A$ is in the bulk of the chain or whether it touches the boundary. The entanglement entropy $S_{A}$ is obtained by determining the number of singlets between $A$ and $B$ for each realization of the disorder and then by multiplying the average by $\ln 2$. We report the variance of the distribution as an estimate of statistical error.

Numerical SDRG results for $S_{A}$ are shown in Fig. 3 (a)(d). In all panels the data are for chains of length $L=$ 1000 and $L=2000$. The disorder average is over 73000 disorder realizations. We start by considering in panel (a) the block $A$ in a periodic chain. For $\ell \lesssim L / 2, S_{A}$ increases logarithmically as function of the interval length $\ell$ (note the logarithmic scale on the $x$-axis). Instead, for $\ell \gtrsim L / 2$ finite-size effects are visible. The dash-dotted line in the 

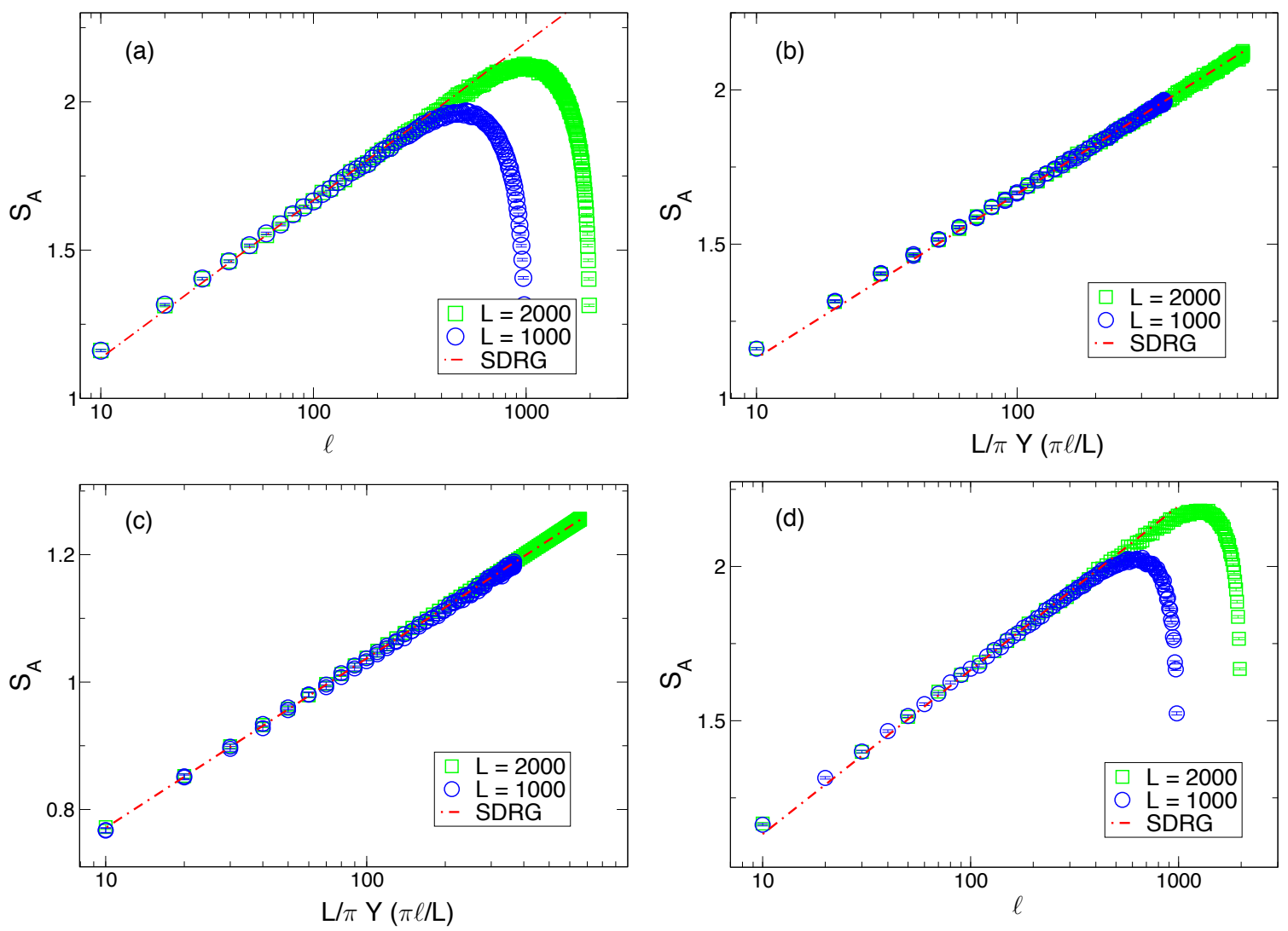

FIG. 3. The single interval entanglement entropy for the disordered Heisenberg spin chain. The data correspond to the average over 73000 disorder realizations. Notice that (here and in the following figures) we report also the statistical errors, although the error bars are smaller than the symbol sizes. Different symbols correspond to different chain sizes $L$ and dashed dotted lines are one-parameter fit to the analytic prediction from the SDRG. (a) A single interval in a periodic chain: $S_{A}$ plotted versus the interval length $\ell$. (b) Same data as in (a) plotted against the modified chord length (44). (c) A single interval starting from the boundary of a chain with OBC versus the modified chord length (44). (d) A single interval in the bulk of an open chain.

figure is a fit to the expected SDRG result ${ }^{49}$

$$
S_{A}=\frac{\ln 2}{3} \ln \ell+K
$$

with $K$ the only fitting parameter. For $\ell \lesssim L / 2$ the data are in perfect agreement with (43).

As discussed in Ref. 52, finite-size effects can be taken into account by replacing in (47) the interval length $\ell$ with the modified chord length $L_{c}$ as

$$
\ell \rightarrow L_{c} \equiv \frac{L}{\pi} Y\left(\frac{\pi \ell}{L}\right) .
$$

Here $Y(x)$ is a symmetric function under $x \rightarrow \pi-x$, which ensures $S(\ell)=S(L-\ell)$. It has been found that for a single block in a periodic chain, $Y(x)$ is well approximated by ${ }^{52}$

$$
Y(x)=\sin (x)\left(1+\frac{4}{3} k_{1} \sin ^{2}(x)\right),
$$

with $k_{1} \sim 0.115$. Using (44) in (43) one obtains

$$
S_{A}=\frac{\ln 2}{3} \ln \left(L_{c}\right)+K \text {. }
$$

Fig. 3 (b) reports the data for $S_{A}$ versus $L_{c}$. The dashdotted line in Fig. 3 (b) is a fit to (46), and it is in perfect agreement with the SDRG data for all values of $\ell$ confirming the correctness of (46).

In Fig. 3 (c) we consider an open chain with the block $A$ starting from the boundary. For a semi-infinite system, the entanglement entropy is expected to be ${ }^{49}$

$$
S_{A}=\frac{\ln 2}{6} \ln (\ell)+K^{\prime},
$$

where the prefactor of the logarithm takes into account that subsystem $A$ shares only one edge with its complement. This formula indeed describes accurately the data for $\ell \lesssim L / 2$. The finite size and boundary effects can be taken into account again by replacing $\ell$ by a modified chord length (44). We find that the same function $Y(x)$ in Eq. (45) describes very accurately the data for OBC as shown in Fig. 3 (c). This is a non trivial result since there is no conformal invariance to guarantee the equality of the two finite size scaling functions (i.e. open and periodic) as in the clean case.

Finally in Fig. 3 (d) we consider a block of length $\ell$ in the center of an open chain. For $\ell \lesssim L / 2$ we expect that 

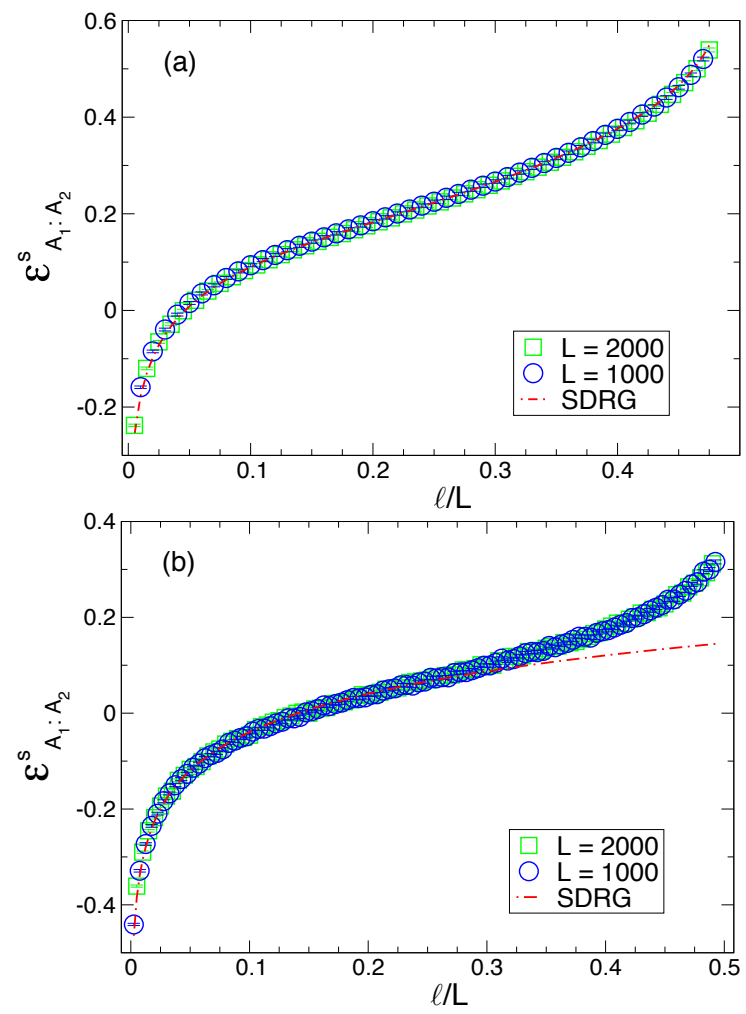

FIG. 4. Shifted logarithmic negativity $\mathcal{E}_{A_{1}: A_{2}}^{s}$ (48) between two adjacent intervals of equal-length $\ell$ in the random Heisenberg chain. $\mathcal{E}_{A_{1}: A_{2}}^{s}$ is plotted against $\ell / L$. The symbols correspond to the average over 73000 disorder realizations. Different symbols correspond to different system sizes. (a): For a periodic chain the data are perfectly described by (49) with only one fitting parameter. (b): For an open chain, we can only use the prediction for the infinite chain which describes well the data as long as $\ell \ll L$.

the block $A$ does not feel significantly the presence of the boundary and (43) should describe accurately the data, as evident from the figure. It is instead not obvious how to modify the prediction to take into account finite-size and boundary effects: we only mention that replacing $\ell$ with the modified chord length (45) does not work. This does not come unexpected since also in clean systems the results is more complicated ${ }^{86}$.

\section{Logarithmic negativity: Two adjacent intervals}

Using the numerical SDRG method, we now study the scaling behavior of the logarithmic negativity $\mathcal{E}_{A_{1}: A_{2}}$ between two adjacent intervals $A_{1}$ and $A_{2}$ in the random Heisenberg chain. Specifically, we provide robust numerical evidence for (41). The negativity is just obtained from the statistics of the singlets between $A_{1}$ and $A_{2}$ and multiplying the resulting average by $\ln 2$.

In Fig. 4 we report the SDRG data for $\mathcal{E}_{A_{1}: A_{2}}$ for two adjacent intervals of the same length $\ell$. Fig. 4 (a) shows the SDRG results for a periodic chain, while Fig. 4 (b) is for two intervals in the middle of an open chain. We plot the shifted negativity

$$
\mathcal{E}_{A_{1}: A_{2}}^{s}=\mathcal{E}_{A_{1}: A_{2}}-\frac{\ln 2}{6} \ln L,
$$

as a function of $\ell / L$ so that data for different chain lengths are expected to collapse on a single scaling curve. This clearly happens for both open and periodic chains. In both cases $\mathcal{E}_{A_{1}: A_{2}}$ increases logarithmically for $\ell \lesssim$ $L / 4$, when finite-size effects kick in. Actually, for PBC one can take into account all the finite-size effects by replacing all lengths in (3) with modified chord lengths, obtaining

$$
\mathcal{E}_{A_{1}: A_{2}}^{s} \simeq \frac{\ln 2}{6} \ln \frac{Y_{c}^{2}(\pi \ell / L)}{Y_{c}(2 \pi \ell / L)}+k .
$$

The SDRG are perfectly described by this prediction as clear from Fig. 4 (a) where the dashed line is a one parameter fit to (49).

Conversely, for the OBC chain in Fig. 4 (b) there is not a simple modification of the result for infinite chain to take into account the boundary effects, as it was the case also for clean systems ${ }^{23}$. For this reason, in the figure we only report the one-parameter fit to the SDRG prediction for the infinite chain (41) valid for $\ell \ll L$.

\section{Logarithmic negativity: Two disjoint intervals}

We finally move to the most interesting case of the logarithmic negativity between two disjoint intervals of equal length $\ell$ at distance $r$. As usual in this section, we compute the negativity from the statistics of the singlets between the two intervals. Our results are reported in Fig. 5 for both periodic and open chains.

In the case of a periodic chain, we plot $\mathcal{E}_{A_{1}: A_{2}}$ as a function of the cross-ratio $x$ (5) in which we have substituted all lengths by the corresponding modified chord lengths, i.e.

$$
x=\frac{Y_{c}^{2}(\pi \ell / L)}{Y_{c}^{2}(\pi(\ell+r) / L)},
$$

in order to take into account finite-size effects. The SDRG asymptotic prediction for the negativity is given by Eq. (4) which reads

$$
\mathcal{E}_{A_{1}: A_{2}}=-\frac{\ln 2}{6} \ln (1-x) .
$$

In Fig. 5 (a) the data correspond to a fixed choice of $r$ and $\ell$ is running up to $(L-r) / 2$. It is clear from the figure that the prediction (51) describes incredibly well the numerical data, without any fitting parameter. Notice that $x$ close to 0 corresponds to far away intervals and the decay of the negativity is algebraic, in constrast with the exponential behavior of clean systems ${ }^{11,12}$. Oppositely, the limit $x \rightarrow 1$ corresponds to very close intervals 

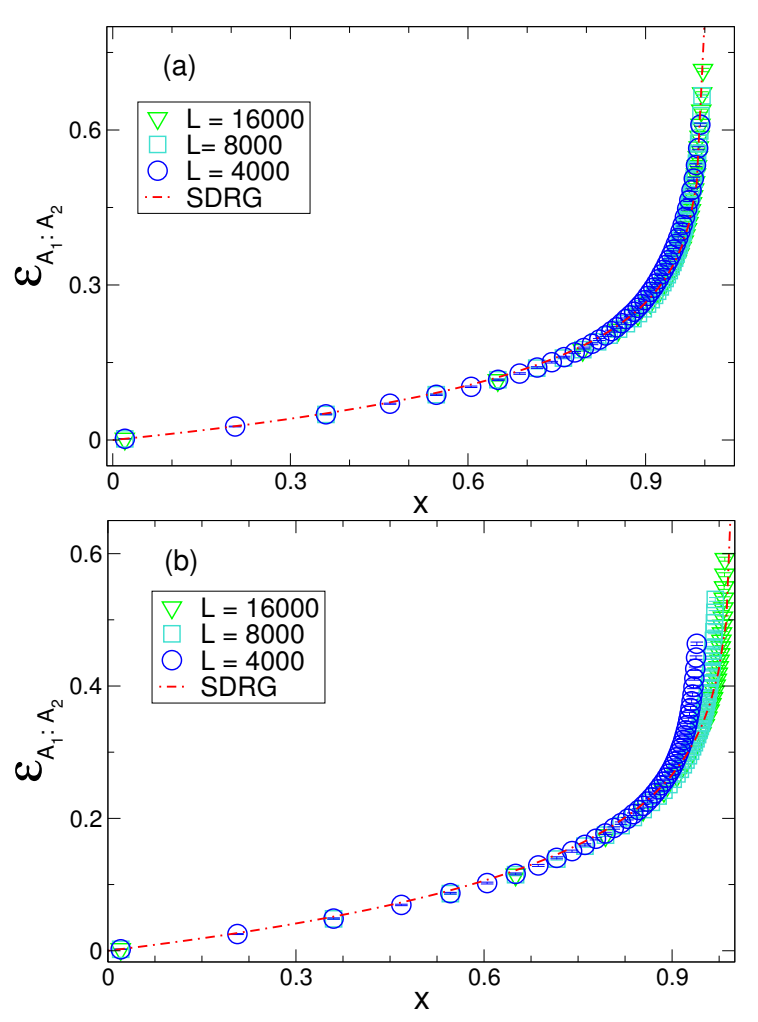

FIG. 5. Logarithmic negativity $\mathcal{E}_{A_{1}: A_{2}}$ between two disjoint intervals of equal length $\ell$ in the random Heisenberg spin chain plotted against the cross ratio $x$. The data are averaged over 73000 disorder realizations. Different symbols correspond to different system sizes. (a): For a periodic chain the crossratio $x$ is given by (50) and the dashed line is the analytic SDRG prediction (51). (b): For an open chain we consider the infinite volume definition of the cross ratio (5) and the dash-dotted line is the SDRG prediction (51) to which the data tend for large chains.

and the logarithmic divergence is needed to reproduce the adjacent intervals results.

For the case of an open chain, we limit ourselves to consider two intervals centered around the middle of the chain, i.e. $A_{1}=[-\ell-r / 2,-r / 2]$ and $A_{2}=[\ell+r / 2, r / 2]$. We generate the data by fixing a value of $r$ and letting $\ell$ run up to the boundary. In this case, we do not have a prediction which takes into account finite size effects, so we expect Eq. (51) to describe correctly the data for $\ell, r \ll L$, i.e. when the effects of the boundary can be neglected. For this reason in Fig. 5 (b) we report the negativity as function of the cross-ratio $x$ given by the infinite volume formula $x=(\ell /(\ell+r))^{2}$. It is evident from the figure that this prediction describes correctly the data for $x$ far from 1 , as it should. With increasing $L$, the effects of the boundaries becomes less and less relevant and the data get closer to the infinite volume result, as expected. (a)

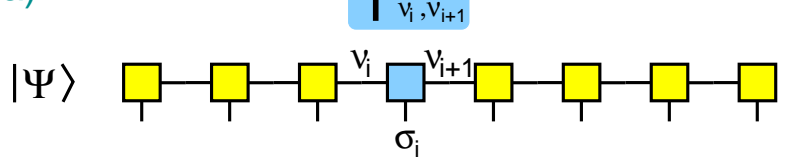

(b)

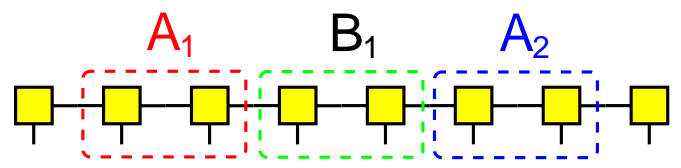

(c)

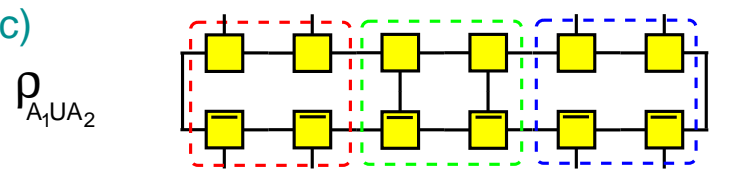

(d)

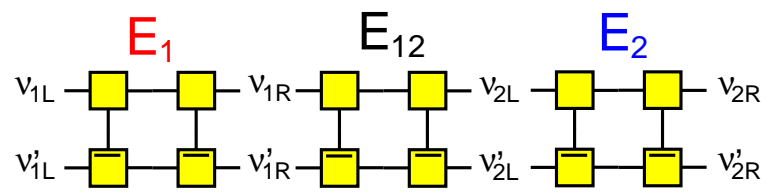

FIG. 6. Reduced density matrix with Matrix Product States (MPS) for a chain with $L=8$ sites and open boundary conditions. (a) The MPS representation for a generic wavefunction $|\Psi\rangle$. The basic tensor $T_{\nu_{i}, \nu_{i+1}}^{\sigma_{i}}$ at site $i$ of the chain is shown in the shaded box. The vertical leg denotes the physical in$\operatorname{dex} \sigma_{i}$, while the horizontal ones are the virtual indices $\nu_{i}$ (summed over). Tensors in the bulk have rank 3, while at the edges have rank 2. (b) The tripartition of the chain as $A_{1} \cup B_{1} \cup A_{2} \cup B_{2} . A_{1}$ and $A_{2}$ are two equal blocks formed by two spins. The two blocks are at distance two (spins in $B_{1}$ ). (c) The reduced density matrix $\rho_{A_{1} \cup A_{2}}$. Only the tensors living in $A_{1} \cup B_{1} \cup A_{2}$ appear. (d) Definition of the transfer matrices $E_{1}, E_{2}, E_{12}$ corresponding to parts $A_{1}, A_{2}$, and $B_{1}$ of the chain.

\section{LOGARITHMIC NEGATIVITY IN DMRG SIMULATIONS}

In this section we describe how to calculate the logarithmic negativity in DMRG simulations. We consider a generic $1 D$ lattice of $L$ sites, restricting ourselves to open boundary conditions. On each site $i$ of the lattice we consider a local Hilbert space of finite dimension $d$ (e.g., for spin- $1 / 2$ systems one has $d=2$ ). A generic wavefunction $|\Psi\rangle$ of a pure state is written as a Matrix-Product-State (MPS) as

$$
|\Psi\rangle=\sum_{\sigma_{1}, \ldots, \sigma_{L}} T_{\nu_{1}}^{\sigma_{1}} T_{\nu_{1} \nu_{2}}^{\sigma_{2}} \cdots T_{\nu_{L}}^{\sigma_{L}}\left|\sigma_{1}, \ldots, \sigma_{L}\right\rangle .
$$

Here $\sigma_{i}$ with $1 \leq i \leq d$ is the so-called physical index representing the states in the local Hilbert space, whereas $1 \leq \nu_{i} \leq \chi_{i}$ are the virtual indices, with $\chi_{i}$ the local bond dimension of the MPS. In (52) the sum over the repeated indices $\nu_{i}$ is assumed, and one has $\left|\sigma_{1}, \sigma_{2}, \ldots, \sigma_{L}\right\rangle \equiv\left|\sigma_{1}\right\rangle \otimes\left|\sigma_{2}\right\rangle \cdots\left|\sigma_{L}\right\rangle$, with $\left|\sigma_{i}\right\rangle$ an element of the basis of the Hilbert space at site $i$. At fixed $\sigma_{i}, T_{\nu_{i} \nu_{i+1}}^{\sigma_{i}}$ is a $\chi_{i} \times \chi_{i}$ matrix, while $T_{\nu_{1}}^{\sigma_{1}}$ and $T_{\nu_{L}}^{\sigma_{L}}$ are vectors. The MPS in (52) can be pictorially represented as 


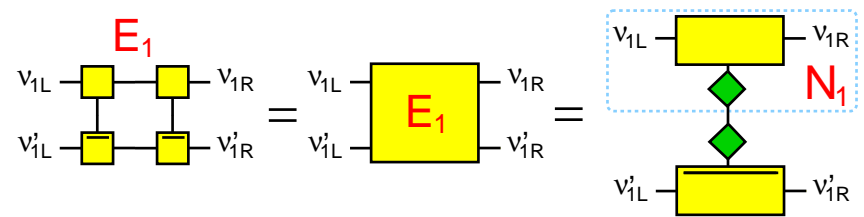

FIG. 7. The definition of the tensor $N_{1}$ (cf. (67)) from the singular value decomposition (SVD) of the transfer matrix $E_{1}$. The SVD is performed with respect to the index pairs $\left(\nu_{1 L}, \nu_{1 R}\right)$ and $\left(\nu_{1 L}^{\prime}, \nu_{1 R}^{\prime}\right)$. The rhombi denote the diagonal matrix containing the square root of the singular values. The same definition holds for $N_{2}$.

in Fig. 6 (a), where the boxes denote the tensors $T_{\nu_{i}, \nu_{i+1}}^{\sigma_{i}}$, and horizontal and vertical legs the virtual and physical indices, respectively. The full system density matrix $\rho \equiv|\Psi\rangle\langle\Psi|$ is obtained as

$$
\begin{aligned}
\rho= & \sum_{\substack{\sigma_{1}, \ldots, \sigma_{L} \\
\sigma_{1}^{\prime}, \ldots, \sigma_{L}^{\prime}}}\left(T_{\alpha_{1}}^{\sigma_{1}} \bar{T}_{\nu_{1}^{\prime}}^{\sigma_{1}^{\prime}}\right)\left(T_{\nu_{1}, \nu_{2}}^{\sigma_{2}} \bar{T}_{\nu_{1}^{\prime}, \nu_{2}^{\prime}}^{\sigma_{2}^{\prime}}\right) \cdots \\
& \cdots\left(T_{\nu_{L}}^{\sigma_{L}} \bar{T}_{\nu_{L}^{\prime}}^{\sigma_{L}^{\prime}}\right)\left|\sigma_{1}\right\rangle\left\langle\sigma_{1}^{\prime}|\otimes \cdots \otimes| \sigma_{L}\right\rangle\left\langle\sigma_{L}^{\prime}\right|,
\end{aligned}
$$

where the bar in $\bar{T}_{\nu_{i}, \nu_{i+1}}^{\sigma_{i}}$ (and correspondingly in the boxes in Fig. 6) denotes the complex conjugation.

\section{A. The partially transposed reduced density matrix}

In order to calculate the logarithmic negativity we now introduce a tripartition of the system (Fig. 6) (b) as $A_{1} \cup B_{1} \cup A_{2} \cup B_{2}$, where $A_{1}$ and $A_{2}$ are the intervals of interest and $B_{1}, B_{2}$ the remaining parts. For simplicity we consider the case of two equal-length intervals with $\ell_{1}=\ell_{2}=\ell$ at mutual distance $r$, and shifted from the left boundary by $s$ sites, i.e., $A_{1}=[s+1, \ldots, s+\ell]$, $A_{2}=[s+\ell+r+1, \ldots, s+r+2 \ell]$. This is illustrated in Fig. 6 (b) for $s=1, \ell=2$, and $d=2$. The reduced density matrix $\rho_{A_{1} \cup A_{2}}$ is obtained by tracing over the degrees of freedom of $B_{1} \cup B_{2}$ in (53) as

$$
\begin{aligned}
\rho_{A_{1} \cup A_{2}}= & \delta_{\nu_{1 L}, \nu_{1 L}^{\prime}} \delta_{\nu_{2 R}, \nu_{2 R}^{\prime}}^{\prime} \\
\times & \times \sum_{\left\{\sigma_{i}, \sigma_{i}^{\prime}: i \in A_{1}\right\}}\left[F_{1}\right]_{\nu_{1 L}, \nu_{1}}^{\left\{\sigma_{i}\right\}}\left[\bar{F}_{1}\right]_{\nu_{1 L}^{\prime}, \nu_{1 R}^{\prime}}^{\left\{\sigma^{\prime}\right\}} \\
\times & \sum_{\left\{\sigma_{i}, \sigma_{i}^{\prime}: i \in A_{2}\right\}}\left[F_{2}\right]_{\nu_{2 L}, \nu_{2 R}}^{\left\{\sigma_{i}\right\}}\left[\bar{F}_{2}\right]_{\nu_{2 L}^{\prime}, \nu_{2 R}^{\prime}}^{\left\{\sigma_{i}^{\prime}\right\}} \\
& \times\left[E_{12}\right]_{\nu_{1 R}^{\prime}, \nu_{2 L}^{\prime}}^{\nu_{1 R}, \nu_{2 L}} \prod_{i \in A_{1} \cup A_{2}}\left|\sigma_{i}\right\rangle\left\langle\sigma_{i}^{\prime}\right|,
\end{aligned}
$$

where we defined

$$
\left[F_{1}\right]_{\nu_{1 L}, \nu_{1 R}}^{\left\{\sigma_{i}\right\}} \equiv \prod_{i \in A_{1}} T_{\nu_{i}, \nu_{i+1}}^{\sigma_{i}},
$$

with $\nu_{1 L} \equiv \nu_{s+1}, \nu_{1 R} \equiv \nu_{s+\ell+1}$ (cf. Fig. 6 (d) for the definition of the indices). A similar definition holds for
$\left[F_{2}\right]_{\nu_{2 L}, \nu_{2 R}}^{\left[\sigma_{i}\right\}}$. The term $\delta_{\nu_{1 L}, \nu_{L}^{\prime}} \delta_{\nu_{2 R}, \nu_{2 R}^{\prime}}$ in (54) is because the virtual indices $\nu_{L}, \nu_{L}^{\prime}$ and $\nu_{1 R}, \nu_{1 R}^{\prime}$ are contracted. In (54) $E_{12}$ is the "transfer matrix" of the region connecting the two intervals $\left(B_{1}\right.$ in Fig. $\left.6(\mathrm{c})\right)$. The definition of $E_{12}$ and of the transfer matrices $E_{1}, E_{2}$, which are associated with the two intervals $A_{1}$ and $A_{2}$, are given as

$$
\begin{aligned}
{\left[E_{1}\right]_{\nu_{1 L}^{\prime}, \nu_{1 R}^{\prime}}^{\nu_{1}, \nu_{1 R}} } & \equiv \prod_{j \in A_{1}} \sum_{\sigma_{j}} T_{\nu_{j}, \nu_{j+1}}^{\sigma_{j}} \bar{T}_{\nu_{j}^{\prime}, \nu_{j+1}^{\prime}}^{\sigma_{j}} \\
{\left[E_{2}\right]_{\nu_{2 L}^{\prime}, \nu_{2 R}^{\prime}}^{\nu_{2 L}, \nu_{2 R}} } & \equiv \prod_{j \in A_{2}} \sum_{\sigma_{j}} T_{\nu_{j}, \nu_{j+1}}^{\sigma_{j}} \bar{T}_{\nu_{j}^{\prime}, \nu_{j+1}^{\prime}}^{\sigma_{j}} \\
{\left[E_{12}\right]_{\nu_{1 R}^{\prime}, \nu_{2 L}^{\prime}}^{\nu_{1 R}, \nu_{2 L}} } & \equiv \prod_{j \in B_{1}} \sum_{\sigma_{j}} T_{\nu_{j}, \nu_{j+1}}^{\sigma_{j}} \bar{T}_{\nu_{j}^{\prime}, \nu_{j+1}^{\prime}}^{\sigma_{j}} .
\end{aligned}
$$

Notice that all the spin indices are contracted in (56)(57)(58). These definitions are illustrated in Fig. 6 (d).

The computational cost to construct $E_{1}, E_{2}, E_{12}$ is $\mathcal{O}\left(\chi_{\max }^{6}\right)$, with $\chi_{\max } \equiv \max _{i}\left(\chi_{i}\right)$ the largest bond dimension of the MPS. It is convenient to introduce a basis for the Hilbert space of the two intervals $A_{1}$ and $A_{2}$ as

$$
\begin{aligned}
\left|w_{\nu_{1}, \nu_{1}}^{(1)}\right\rangle & \equiv \sum_{\sigma_{i}: i \in A_{1}}\left[F_{1}\right]_{\nu_{1 L}, \nu_{1 R}}^{\left\{\sigma_{i}\right\}} \prod_{j \in A_{1}}\left|\sigma_{j}\right\rangle, \\
\left|w_{\nu_{2 L}, \nu_{2}}^{(2)}\right\rangle & \equiv \sum_{\sigma_{i}: i \in A_{2}}\left[F_{2}\right]_{\nu_{2 L}, \nu_{2 R}}^{\left\{\sigma_{i}\right\}} \prod_{j \in A_{2}}\left|\sigma_{j}\right\rangle,
\end{aligned}
$$

where the index pairs $\left(\nu_{1 L}, \nu_{1 R}\right)$ and $\left(\nu_{2 L}, \nu_{2 R}\right)$ label the different states in the two bases. This allows one to rewrite (54) in the compact form

$$
\begin{aligned}
& \rho_{A_{1} \cup A_{2}}=\delta_{\nu_{1 L}, \nu_{1 L}^{\prime}} \delta_{\nu_{2 R}, \nu_{2 R}^{\prime}}\left[E_{12}\right]_{\nu_{1 R}^{\prime}, \nu_{2 L}^{\prime}}^{\nu_{1 R}, \nu_{2 L}} \\
& \left|w_{\nu_{1 L}, \nu_{1 R}}^{(1)}\right\rangle\left\langle w_{\nu_{1 L}^{\prime}, \nu_{1 R}^{\prime}}^{(1)}|\otimes| w_{\nu_{2 L}, \nu_{2 R}}^{(2)}\right\rangle\left\langle w_{\nu_{2 L}^{\prime}, \nu_{2 R}^{\prime}}^{(2)}\right| .
\end{aligned}
$$

Notice that now $\rho_{A_{1} \cup A_{2}}$ is at most a $\chi_{\max }^{2} \times \chi_{\max }^{2}$ matrix. Importantly, for a generic tripartition the vectors $\left|w_{\nu_{1}, \nu_{1}}^{(1)}\right\rangle$ and $\left|w_{\nu_{2 L}, \nu_{2 R}}^{(2)}\right\rangle$ are not orthogonal. Clearly, one has the overlap matrices

$$
\begin{aligned}
& \left\langle w_{\nu_{1 L}, \nu_{1 R}}^{(1)} \mid w_{\nu_{1 L}^{\prime}, \nu_{1 R}^{\prime}}^{(1)}\right\rangle=\left[E_{1}\right]_{\nu_{1 L}^{\prime}, \nu_{1 R}^{\prime}}^{\nu_{1 L}, \nu_{R}} \\
& \left\langle w_{\nu_{2 L}, \nu_{2 R}}^{(2)} \mid w_{\nu_{2 L}^{\prime}, \nu_{2 R}^{\prime}}^{(2)}\right\rangle=\left[E_{2}\right]_{\nu_{2 L}^{\prime}, \nu_{2 R}^{\prime}}^{\nu_{2 L}, \nu_{2 R}},
\end{aligned}
$$

where $E_{1}$ and $E_{2}$ are defined in (56) and (57), respectively.

Interestingly, when the the two intervals $A_{1}$ and $A_{2}$ are close to the edges of the chain (this is the geometry considered in Ref. 17) one has that $\left|w_{\nu_{1 L}, \nu_{1 R}}^{(1)}\right\rangle \rightarrow\left|w_{\nu_{1 R}}^{(1)}\right\rangle$ and $\left|w_{\nu_{2 L}, \nu_{2}}^{(2)}\right\rangle \rightarrow\left|w_{\nu_{2 L}}^{(2)}\right\rangle$, with $\left|w_{\nu_{1}}^{(1)}\right\rangle$ and $\left|w_{\nu_{2 L}}^{(2)}\right\rangle$ forming two orthonormal bases. Thus, it is straigthforward to show that $\rho_{A_{1} \cup A_{2}}$ is simply given as

$$
\rho_{A_{1} \cup A_{2}}=\left[E_{12}\right]_{\nu_{1}^{\prime}, \nu_{2 L}^{\prime}}^{\nu_{1 R}, \nu_{2 L}}\left|w_{\nu_{1 R}}^{(1)}\right\rangle\left\langle w_{\nu_{1 R}^{\prime}}^{(1)}|\otimes| w_{\nu_{2 L}}^{(2)}\right\rangle\left\langle w_{\nu_{2 L}^{\prime}}^{(2)}\right| .
$$

The spectrum of $\rho_{A_{1} \cup A_{2}}$ is obtained by diagonalizing $E_{12}$, with computational cost $\propto \chi_{\max }^{6}$. Moreover, $\rho_{A_{1} \cup A_{2}}^{T_{2}}$ is obtained from (64) by exchanging $\nu_{2 L} \leftrightarrow \nu_{2 L}^{\prime}$. 
(a)
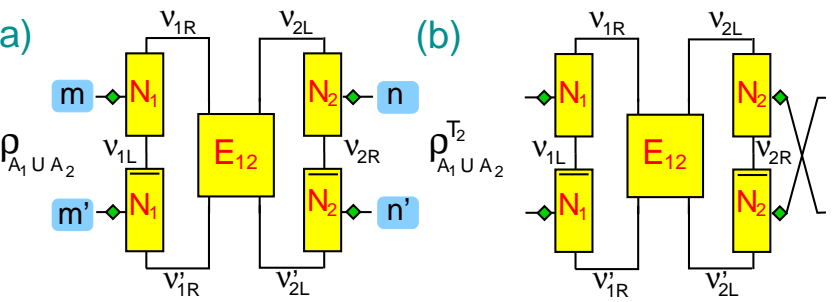

FIG. 8. (a) The reduced density matrix of two intervals $\rho_{A_{1} \cup A_{2}}$ in the MPS framework. The tensor $N_{1}$ is defined in Fig. 7 (a similar definition holds for $N_{2}$ ), whereas $E_{12}$ is as in Fig. 6 (d). The indices are as in (67). The index pairs $\left(m, m^{\prime}\right)$ and $\left(n, n^{\prime}\right)$ refer to interval $A_{1}$ and $A_{2}$, respectively. (b) The partially transposed reduced density matrix $\rho_{A_{1} \cup A_{2}}^{T_{2}}$. Here the partial transposition is performed with respect to subsystem $A_{2}$, and it corresponds to the exchange $n \leftrightarrow n^{\prime}$.

For generic tripartitions it is convenient to orthonormalize the vectors $\left|w_{\nu_{1 L}, \nu_{1 R}}^{(1)}\right\rangle$ and $\left|w_{\nu_{2 L}, \nu_{2} R}^{(2)}\right\rangle$. This can be done via a singular value decomposition (SVD) of the transfer matrices $E_{1}$ and $E_{2}$ (cf. (56)(57)). Precisely, given the SVD of $E_{1}$ as $E_{1}=U D V^{\dagger}$ with $U, V$ unitaries and $D$ diagonal, the vectors $\left|v_{m}^{(1)}\right\rangle$ defined as

$$
\left|v_{m}^{(1)}\right\rangle \equiv\left[\widetilde{N}_{1}\right]_{\nu_{1 L}, \nu_{1 R}}^{m}\left|w_{\nu_{1 L}, \nu_{1 R}}^{(1)}\right\rangle
$$

with $\widetilde{N}_{1} \equiv D^{-\frac{1}{2}} U^{\dagger}$, are orthonormal. Similarly, for $A_{2}$ one has the orthonormal vectors

$$
\left|v_{m}^{(2)}\right\rangle \equiv\left[\widetilde{N}_{2}\right]_{\nu_{2 L}, \nu_{2 R}}^{m}\left|w_{\nu_{2 L}, \nu_{2 R}}^{(2)}\right\rangle .
$$

Using (65) and (66) one can rewrite $\rho_{A_{1} \cup A_{2}}$ as

$$
\begin{aligned}
& \rho_{A_{1} \cup A_{2}}= \\
& \quad\left(\left[N_{1}\right]_{\nu_{1 L}, \nu_{1 R}}^{m}\left[\bar{N}_{1}\right]_{\nu_{1 L}, \nu_{1 R}^{\prime}}^{m^{\prime}}\right)\left(\left[N_{2}\right]_{\nu_{2 L}, \nu_{2 R}}^{n}\left[\bar{N}_{2}\right]_{\nu_{2 L}^{\prime}, \nu_{2 R}}^{n^{\prime}}\right) \\
& \quad \times\left[E_{12}\right]_{\nu_{1 R}^{\prime}, \nu_{2 L}^{\prime}}^{\nu_{1 R}, \nu_{2 L}}\left|v_{m}^{(1)}\right\rangle \otimes\left|v_{n}^{(2)}\right\rangle\left\langle v_{m^{\prime}}^{(1)}\right| \otimes\left\langle v_{n^{\prime}}^{(2)}\right|
\end{aligned}
$$

where $N_{1} \equiv \widetilde{N}_{1}^{-1}=U D^{\frac{1}{2}}$, and similarly for $N_{2}$. The definition of $N_{1}$ from the SVD of $E_{1}$ is illustrated in Fig. 7. Formula (67) is illustrated pictorially in Fig. 8 (a). In (67) all the indices are contracted except for $m, m^{\prime}, n, n^{\prime}$. Precisely, the index pair $\left(m, m^{\prime}\right)$ refers to subsystem $A_{1}$, whereas $\left(n, n^{\prime}\right)$ is associated with $A_{2}$. Moreover, one has that $n, m, n^{\prime}, m^{\prime} \in\left[1, \chi_{\max }^{2}\right]$, implying that the computational cost to evaluate the terms in the round brackets in (67) is $\propto \chi_{\max }^{7}$. However, this can be reduced by considering a truncated SVD of $E_{1}$ and $E_{2}$, i.e., keeping only the $p\left(p \ll \chi_{\max }^{2}\right)$ largest singular values. This relies on the fact that the singular values of $E_{1}$ and $E_{2}$ typically decay very quickly. The computational cost is now $p^{2} \chi_{\max }^{3}$. The same trick is used in the implementation of DMRG with periodic boundary conditions $^{87}$. Finally, from (67) it is straightforward to obtain $\rho_{A_{1} \cup A_{2}}^{T_{2}}$ by exchanging $n \leftrightarrow n^{\prime}$, as illustrated in Fig. 8 (b).

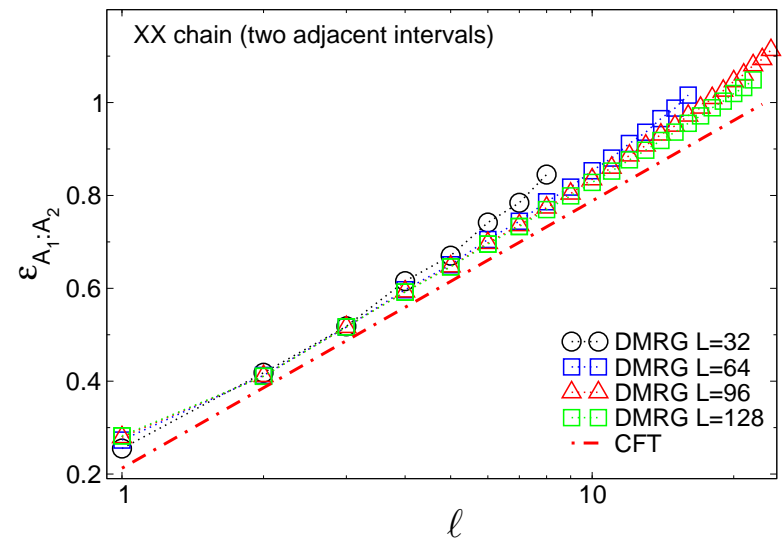

FIG. 9. The logarithmic negativity $\mathcal{E}_{A_{1}: A_{2}}$ between the two (equal-length) adjacent intervals $A_{1} \cup A_{2}$ in the $X X$ chain. Here the two intervals are at the center of the chain. $\mathcal{E}_{A_{1}: A_{2}}$ is plotted against the length of one interval $\ell$. The symbols are DMRG data for a chain with length up to $L=128$. The dash-dotted line is the CFT prediction $1 / 4 \ln \ell$ in the thermodynamic limit.

\section{B. The clean $X X$ chain: Two adjacent intervals}

Before moving to disordered systems it is worth providing some checks of (67) for clean systems in which all results are under control. To this purpose here we focus on the clean $X X$ chain

$$
\mathcal{H}_{X X}=J \sum_{i=1}^{L-1}\left(S_{i}^{x} S_{i+1}^{x}+S_{i}^{y} S_{i+1}^{y}\right)+h \sum_{i=1}^{L} S_{i}^{z},
$$

with open boundary conditions. We fix $J=1$, considering zero magnetic field $h=0$. We restrict ourselves to two adjacent intervals at the center of the chain (see Fig. 1 (a)). This corresponds to the trivial transfer matrix $\left[E_{12}\right]_{\nu_{1 R}^{\prime}, \nu_{2 L}^{\prime}, \nu_{2 L}}^{\nu_{1}}=\delta_{\nu_{1 R} \nu_{2 L}} \delta_{\nu_{1 R}^{\prime} \nu_{2 L}^{\prime}}$ in (67).

DMRG data for the logarithmic negativity $\mathcal{E}_{A_{1}: A_{2}}$ between two adjacent intervals of equal length $\ell$ are reported in Fig. 9. The different symbols correspond to $L=32,64,96,128$. In the DMRG simulations we fixed the largest bond dimension to $\chi_{\max }=80$, and the discarded weight ${ }^{16}$ to $\epsilon \sim 10^{-11}$. Also, to reduce the computational cost, we considered a truncated SVD of the transfer matrices $E_{1}$ and $E_{2}$ (cf. (67) and Fig. 7) fixing $p=200$.

The DMRG data for $\mathcal{E}_{A_{1}: A_{2}}$ are plotted against the length of the two intervals $\ell$ (notice the logarithmic scale on the $x$-axis). There are no CFT predictions for $\mathcal{E}_{A_{1}: A_{2}}$ in finite systems with open boundary conditions, so we can only use the result for two adjacent intervals in the infinite chain $^{11}$

$$
\mathcal{E}_{A_{1}: A_{2}}=\frac{c}{4} \ln \left(\frac{\ell_{1} \ell_{2}}{\ell_{1}+\ell_{2}}\right)+\text { cnst },
$$

with $\ell_{1}$ and $\ell_{2}$ the lengths of the two intervals, $c$ the central charge, and cnst an additive constant. This is 
expected to describe the data for an open chain as long as $\ell_{1}, \ell_{2} \ll L$. This is confirmed for the $X X$ chain by the data in Fig. 9 in which the dash-dotted line is (69) for $\ell_{1}=\ell_{2}=\ell$. While some deviations from (69) are visible for finite $L, \ell$, the agreement with the CFT prediction (69) becomes progressively better upon increasing $L$, in the region $\ell \ll L$.

\section{NEGATIVITY IN THE RANDOM $X X$ CHAIN: SOME DMRG RESULTS}

Here we discuss the logarithmic negativity $\mathcal{E}_{A_{1}: A_{2}}$ of two adjacent intervals in the random $X X$ chain $(\Delta=0$ in (6), see Appendix (A)). To this purpose we perform DMRG simulations, focusing on the disorder distribution $P_{\delta}(J)=\delta^{-1} J^{-1+1 / \delta}$, with $\delta=1 / 4,1,3 / 2$. Increasing values of $\delta$ correspond to increasing disorder strength. Specifically, $\delta=0$ corresponds to the clean case, whereas for $\delta \rightarrow \infty$ one recovers the infinite-randomness fixed point (IRFP) distribution (cf. (16)). Our data for $\mathcal{E}_{A_{1} \cup A_{2}}$ correspond typically to an average over $\sim 10^{3}$ disorder realizations. Although exact results for the negativity are not yet available, many entanglement-related quantities, such as the entanglement entropy, can be calculated for the random $X X$ chain, via a mapping to free fermions (cf. Appendix A). Importantly, this provides a reliable way to check the accuracy of DMRG results.

\section{A. Convergence of DMRG}

For each disorder realization, a crucial aspect is the convergence of the DMRG method. This depends both on the chain sizes $L$ and on the disorder strength $\delta$. Specifically, we numerically observed that the convergence of DMRG becomes rapidly poor upon increasing $L$ or $\delta$. This is physically expected because the disorder gives rise to a "rough" energy landscape, which makes likely for DMRG to get trapped in a local minimum. This affects severely the convergence because typical DMRG update schemes are local ${ }^{16}$. This is also related to the exponential small gap ${ }^{81} \ln \Delta E \sim-L^{1 / 2}$ of the RS phases. To check the correctness of the DMRG results, for each disorder realization we compared the data for the halfchain von Neumann entropy with the exact calculation using free-fermion techniques (see Appendix A). We used as a DMRG convergence criterion the matching of the two results within a precision of $10^{-3}$. We also defined the DMRG convergence rate $R_{c}$ as the fraction of converged DMRG simulations. Only converged disorder configurations were included in the disorder averages.

The fact that $R_{c}<1$ results in a systematic error in the DMRG data. This can be mitigated by using a large number of DMRG sweeps. We typically used $n_{s w} \approx 100$ in our simulations. Moreover, we observed that in each sweep it is crucial to perform a sufficient number of Lanczos iterations $n_{\text {iter }}$ when locally optimizing the MPS. In our simulations we used $n_{\text {iter }} \approx 100$. Still, we should stress that this is not sufficient to ensure $R_{c}=1$. For instance, we observed that for $L=63$ at $\delta=1$, using $n_{s w}=100$ and $n_{\text {iter }}=100$, one has $R_{c} \approx 0.9$. Finally, to provide reliable error bars for our DMRG results, we always checked that the systematic error was negligible compared with the statistical error arising from the disorder average, providing the latter as our final error estimate.

Fig. 10 (a) shows the comparison between the DMRG data for $S_{A}$ (empty symbols in the figure) and the exact results (full symbols) obtained using free-fermion methods (see Appendix A). Data are for $\delta=3 / 2$, which is the most difficult value of $\delta$ to simulate, and $L=16,24$. The error bars are the statistical errors resulting from the disorder average. In all cases the DMRG results are in agreement with the free-fermion result, within error bars. This suggests that, at least for the von Neumann entropy, the systematic error due to $R_{c}<1$ is negligible compared to the statistical one.

\section{B. DMRG results}

Fig. 10 (b) reports the von Neumann entropy $S_{A}$ for the interval $A$ at the center of the open chain. The symbols are DMRG data for different $\delta$ and $L$. The dash-dotted line is the CFT result in the clean case $S_{A} \simeq 1 / 3 \ln \ell+c^{\prime}$, whereas the dotted line is the SDRG result

$$
S_{A} \simeq \frac{\ln 2}{3} \ln \ell+\text { const. }
$$

We recall that the fixed point SDRG is expected to describe the numerical data for any disorder strength, but for asymptotically large $\ell$ and $L$. For finite interval and system lengths, we expect a complicated crossover between the clean and SDRG results, which is more severe for small disorder. In fact, from Fig. 10 (b) it is clear that for the smallest disorder strength $(\delta=1 / 4)$, the data are closer to the clean prediction than to the SDRG one. This does not come unexpected since the crossover between the two fixed points should be regulated by a $\delta$-dependent crossover length $\xi_{\delta}$. One should expect that $\xi_{\delta} \rightarrow \infty$ for $\delta \rightarrow 0$. At any $\delta$, the IRFP results should be valid only for $\ell, L \gg \xi_{\delta}$. However, the data for $\delta=1$ and $\delta=3 / 2$ are almost indistinguishable, signaling that they should be both in the SDRG regime. Indeed, although oscillating corrections are present (as it is well known ${ }^{52}$ ), the data are in rough agreement with the SDRG prediction (70) (dotted line). A more quantitative analysis of the entanglement entropy, with a proper robust determination of the prefactor to the logarithmic growth, requires the study of much longer chains as done by means of free fermionic methods ${ }^{50,52}$. Unfortunately, simulating with DMRG chains of length of the order of hundreds sites and with sufficient statistics is beyond our current capability.

Although the data for the entanglement entropy are asymptotically in rough agreement with the prediction 

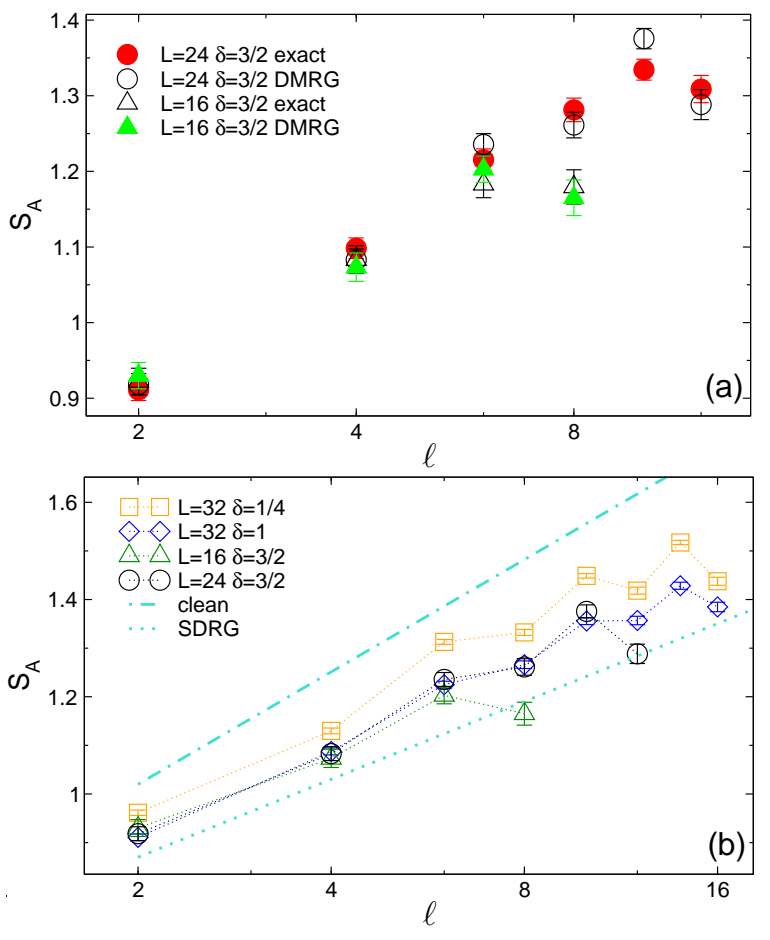

FIG. 10. Entanglement entropy in the random XX spin chain. Panel (a): Check of the DMRG convergence. The von Neumann entropy $S_{A}$ for a single interval at the center of an open chain plotted versus the interval length $\ell$. The empty symbols denote DMRG results for chains with $L=16,24$ and disorder strength $\delta=3 / 2$. The data are averaged over $\sim 10^{3}$ disorder realizations. The full symbols are exact results obtained using free-fermion techniques. Panel (b): Same geometry as in (a). The symbols are DMRG data for $L=16,24,28,32$ and $1 / 4 \leq \delta \leq 3 / 2$. Here $\delta=0$ and $\delta \rightarrow \infty$ correspond to the clean case and the IRFP, respectively. For $\delta=1$ one has the box distribution. The dash-dotted line is the CFT prediction for $\delta=0$. The dotted line is the strong disorder renormalization group (SDRG) prediction in the thermodynamic limit.

of the SDRG already for $L=32$ and $\delta \sim 1$, this does not imply that the same is true for the negativity, because the crossover between the CFT (clean) and the IRFP fixed points in principle depends on the measured quantity. In order to understand the scaling of the negativity, it is worth to consider the Rényi entropy

$$
S_{A}^{(1 / 2)} \equiv 2 \operatorname{Tr} \rho_{A}^{1 / 2},
$$

for a single interval $A$ of length $\ell$ at the center of an open chain. Indeed, the negativity for pure states (e.g., for two adjacent intervals with $\ell_{1}+\ell_{2}=L$ ) coincides with $S_{A}^{(1 / 2)}$. Consequently, one could expect the scaling of the negativity to resemble that of $S_{A}^{(1 / 2)}$, rather than that of the von Neumann entropy $S_{A}$. In Fig. 11 (a) we report exact numerical data for $S_{A}^{(1 / 2)}$ at $\delta=1$ obtained by means of free-fermionic techniques, which allow us to reach large systems sizes $(L=200)$. Interestingly, the data do not show strong parity effects (i.e., oscillations with the parity of the block size), in contrast with $S_{A}$
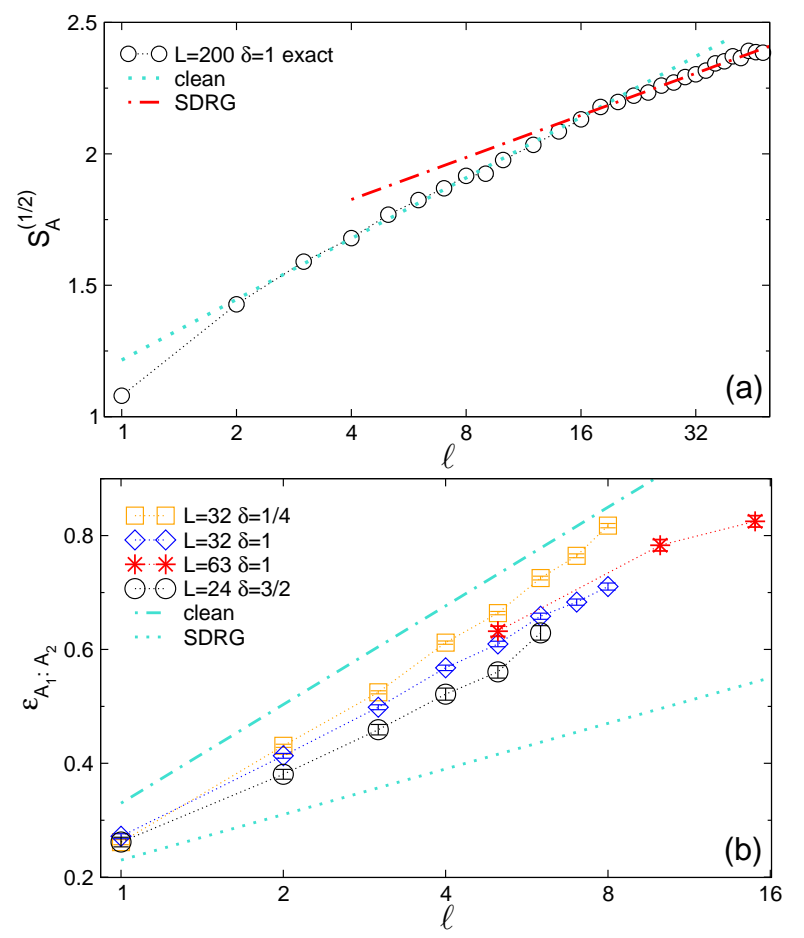

FIG. 11. Entanglement negativity in the random XX spin chain. Panel (a): Rényi entropy $S_{A}^{(1 / 2)}$ for a single interval at the center of an open chain plotted versus the interval length $\ell$. The data (circles) are obtained by means of freefermionic techniques which allow us to reach large systems sizes $(L=200)$. Note the crossover from the scaling of the clean model at short lengths (dotted line) to the SDRG one at large distances (dash-dotted line). Panel (b): The logarithmic negativity $\mathcal{E}_{A_{1}: A_{2}}$ for two adjacent intervals of equal length $\ell$ (in the center of an open chain) plotted as a function of $\ell$. The symbols denote DMRG results for chains with $L=$ $16,24,28,32,63$ and disorder strength $1 / 4 \leq \delta \leq 3 / 2$. The data are averaged over $\sim 10^{3}$ disorder realizations. The dashdotted and dotted lines are the CFT prediction for $\delta=0$, and the SDRG result, respectively.

(the same is true for the negativity in Fig. 11 (b)). Considering these large system sizes we can clearly see the crossover from the scaling of the clean model at short lengths $\left(S_{A}^{(1 / 2)} \sim 1 / 2 \ln \ell\right)$ to the SDRG one at large distances $\left(S_{A}^{(1 / 2)} \sim(\ln 2) / 3 \ln \ell\right)$. The crossover starts around $\ell \sim 15$ and it is fully established around $\ell \sim 30$, implying that $L \approx 120=4 \times 30$ would be needed to fully confirm our results. This, however, is not possible with our current DMRG implementation.

Let us, however, consider the logarithmic negativity $\mathcal{E}_{A_{1}: A_{2}}$ between the two adjacent intervals. The DMRG data are reported in Fig. 11 (b) for chain sizes up to $L=63$ and for several disorder strengths. The dashdotted line is the CFT prediction for the clean model $\mathcal{E}_{A_{1}: A_{2}} \simeq 1 / 4 \ln (\ell)+c^{\prime}$ (cf. Fig. 9) while the dotted line is the SDRG result $\mathcal{E}_{A_{1}: A_{2}} \simeq(\ln 2) / 6 \ln (\ell)+$ const (cf. (41)). For $\delta=1 / 4, \mathcal{E}_{A_{1} \cup A_{2}}$ grows with a slope simi- 
lar to the clean case (compare also with Fig. 9). This does not come unexpected since also the results for the entanglement entropy in Fig. 10 (b) for $\delta=1 / 4$ are affected by a strong crossover. Increasing the strength of the disorder, we observe that for the available subsystems' sizes the slopes of the negativity appears to reduce, but, as a difference with the entanglement entropy, there is no saturation with $\delta$ and also for $\delta=3 / 2$ there are still strong crossover effects. This is in complete analogy with what observed for $S_{A}^{(1 / 2)}$ in Fig. 11 (a), where the crossover for $\delta=1$ starts taking place around $\ell=15$. Indeed, for $\delta=1$, the last points for $L=63$ with $\ell=15$ seem to move in the right direction (we cannot access chains of this length for $\delta=3 / 2$ because of the poor convergence of DMRG for this large disorder). However, these are only very encouraging signals for the correctness of our prediction: we can only conclude that for the system sizes and $\delta$ accessible to DRMG simulations, $\mathcal{E}_{A_{1}: A_{2}}$ exhibits deviations from the SDRG prediction (41), which are likely explained as crossover effects. Much larger chain sizes (of the order of $L \sim 120 / 130$ which are not accessible with our current DMRG implementation), would be needed for the asymptotic IRFP behavior to set in.

\section{DISCUSSION}

In this manuscript we considered the scaling behavior of the logarithmic negativity of disordered spin chains in the random singlet phase. Our main results have been already summarized in the Introduction, and for this reason here we only mention some new research directions originating from the present work. First, in the random $X X$ chain it should be possible by generalizing the results for clean systems ${ }^{25,36,37}$ to treat the moments of the partially-transposed density matrix exactly. By comparing with the analytic results of section III A, this would allow to provide a more robust check of some of our findings. Another intriguing direction would be to investigate to which extent the relation between the negativity and the mutual information remains valid in more complicated disordered phases. In this respect one possibility would be to focus on the spin-1 random Heisenberg chain $^{56}$. Different physical behavior should also appear in the spin-1/2 Heisenberg chain in which, besides antiferromagnetic couplings, ferromagnetic ones are allowed. Finally, it would be interesting to investigate the scaling of the negativity in excited states of disordered spin models, also in connection with many-boby localization.

\section{ACKNOWLEDGMENTS}

We acknowledge very useful discussions with G. Sierra and K. Agarwal. We also would like to thank M. Collura, F. Pollmann and M. Stoudenmire for very useful insights on the DMRG simulations, and on the ITensor library.
All the authors acknowledge support from the ERC under the Starting Grant 279391 EDEQS.

\section{Appendix A: The disordered $X X$ chain}

The random $X X$ chain with open boundary conditions is defined by the Hamiltonian

$$
\mathcal{H}_{X X}=\sum_{i=1}^{L-1} J_{i}\left(S_{i}^{x} S_{i+1}^{x}+S_{i}^{y} S_{i+1}^{y}\right)+h \sum_{i=1}^{L} S_{i}^{z},
$$

with $S_{i}^{x, y, z} \equiv \sigma_{i}^{x, y, z} / 2, \sigma_{i}^{\alpha}$ being the Pauli matrices acting on site $i$. For periodic boundary conditions one has an extra term in Eq. (A1) connecting site $L$ with site 1 . Hereafter we fix $h=0$. Here $J_{i}$ are uncorrelated random variables. After the Jordan-Wigner transformation

$$
c_{i}=\left(\prod_{m=1}^{i-1} \sigma_{m}^{z}\right) \frac{\sigma_{i}^{x}-i \sigma_{i}^{y}}{2}
$$

(A1) is recast in the free-fermionic form

$$
\mathcal{H}_{X X}=\frac{1}{2} \sum_{i=1}^{L-1} J_{i}\left(c_{i}^{\dagger} c_{i+1}+c_{i+1}^{\dagger} c_{i}\right)+\frac{h}{2} \sum_{i=1}^{L-1} c_{i}^{\dagger} c_{i}
$$

with $c_{i}$ spinless fermionic operators satisfying the canonical anticommutation relations $\left\{c_{m}, c_{n}^{\dagger}\right\}=\delta_{m, n}$.

One can now impose that the single-particle eigenstates $\left|\Psi_{q}\right\rangle$ (with $q$ an integer labelling the different eigenstates) of (A3) are of the form

$$
\eta_{q}^{\dagger}|0\rangle \equiv\left|\Psi_{q}\right\rangle=\sum_{i} \Phi_{q}(i) c_{i}^{\dagger}|0\rangle
$$

with $|0\rangle$ the fermion vacuum, $\Phi_{q}(i)$ the eigenstate amplitudes, and $\eta_{q}$ new fermionic operators. The Schrödinger equation, which allows to determine $\Phi_{q}(i)$, becomes

$$
J_{i} \Phi_{q}(i+1)+J_{i-1} \Phi_{q}(i-1)=2 \epsilon_{q} \Phi_{q}(i), \quad \forall i,
$$

with $J_{L}=0$, and $\epsilon_{q}$ the single-particle eigenvalues. Notice that (A5) is the eigenvalue problem for the banded matrix $T \equiv\left(J_{j} \delta_{i, j+1}+J_{j-1} \delta_{i, j-1}\right) / 2$. One can show that the eigenvalues of the matrix $T$ are organized in pairs with opposite sign. Precisely, given the amplitude $\Phi_{1}(i)$ of an eigenvector with $\epsilon_{q}>0$, the amplitudes of the eigenvector with eigenvalue $-\epsilon_{q}$ are obtained as $(-1)^{i+1} \Phi_{q}(i)$. As a consequence, the ground state $|G S\rangle$ of (A1) is in the sector with $M=L / 2$ fermions. This is constructed by filling all the negative modes $\epsilon_{q}<0$ as

$$
|G S\rangle=\eta_{q_{M}}^{\dagger} \eta_{q_{M-1}}^{\dagger} \cdots \eta_{q_{1}}^{\dagger}|0\rangle
$$

It is straightforward to derive the anticommutation relations

$$
\left\{\eta_{q}^{\dagger}, c_{j}^{\dagger}\right\}=\left\{\eta_{q}, c_{j}\right\}=0
$$


and

$$
\left\{\eta_{q}^{\dagger}, c_{j}\right\}=\Phi_{q}(j) \delta_{k, j}, \quad\left\{\eta_{q}, c_{j}^{\dagger}\right\}=\Phi^{*}(j) \delta_{k, j} .
$$

Using (A7) and (A8), the expectation value of the twopoint function $\left\langle c_{i}^{\dagger} c_{i}\right\rangle$ in a generic eigenstate of (A1) is given as

$$
\left\langle c_{i}^{\dagger} c_{j}\right\rangle=\sum_{q} \Phi_{q}^{*}(i) \Phi_{q}(j),
$$

where the sum if over the $q$ single-particle excitations forming the eigenstate.

In order to calculate the entanglemen entropy, let us now introduce a bipartition of the chain into two subsystems as $A \cup \bar{A}$, with $\bar{A}$ denoting the complement of $A$. For freefermion models, even in presence of disorder, the reduced density matrix $\rho_{A}$ of subsystem $A$ is determined by the correlation matrix ${ }^{88-93}$ restricted to $A$ as

$$
\mathcal{C}_{i j}^{(A)} \equiv\left\langle c_{i}^{\dagger} c_{j}\right\rangle
$$

where $i, j \in A$. In particular, given the eigenvalues $\lambda_{k}$ of $\mathcal{C}^{(A)}$, the entanglement entropy $S_{A}$ is given as

$$
S_{A}=-\sum_{k}\left(\lambda_{k} \ln \lambda_{k}+\left(1-\lambda_{k}\right) \ln \left(1-\lambda_{k}\right)\right) .
$$

1 L. Amico, R. Fazio, A. Osterloh, and V. Vedral, Rev. Mod. Phys. 80, 517 (2008).

2 P. Calabrese, J. Cardy, and B. Doyon Eds, J. Phys. A 42, 500301 (2009).

3 J. Eisert, M. Cramer, and M. B. Plenio, Rev. Mod. Phys. 82, 277 (2010).

4 N. Laflorencie, arXiv:1512.03388.

5 A. Peres, Phys. Rev. Lett. 77, 1413 (1996).

${ }^{6}$ K Zyczkowski, P. Horodecki, A. Sanpera, and M. Lewenstein, Phys. Rev. A 58, 883 (1998).

7 K. Zyczkowski, Phys. Rev. A 60, 3496 (1999).

8 J. Lee, M. S. Kim, Y. J. Park, and S. Lee, J. Mod. Opt. 47, 2151 (2000); J. Eisert and M. B. Plenio, J. Mod. Opt. 46, 145 (1999).

${ }^{9}$ G. Vidal and R. F. Werner, Phys. Rev. A 65, 032314 (2002).

10 M. B. Plenio, Phys. Rev. Lett. 95, 090503 (2005); J. Eisert, quant-ph/0610253.

11 P. Calabrese, J. Cardy, and E. Tonni, Phys. Rev. Lett. 109, 130502 (2012).

12 P. Calabrese, J. Cardy, and E. Tonni, J. Stat. Mech. P02008 (2013).

13 P. Calabrese, L. Tagliacozzo, and E. Tonni, J. Stat. Mech. (2013) P05002.

14 S. White, Phys. Rev. Lett. 69, 2863 (1992).

15 U. Schollwöck, Rev. Mod. Phys. 77, 259 (2005).

16 U. Schollwöck, Annals of Physics 326, 96 (2011).

17 H. Wichterich, J. Molina-Vilaplana, and S. Bose, Phys. Rev. A 80, 010304 (2009).

18 S. Marcovitch, A. Retzker, M. B. Plenio and B. Reznik, Phys. Rev. A 80, 012325 (2009).

19 H. Wichterich, J. Vidal, and S. Bose, Phys. Rev. A 81, 032311 (2010).

${ }^{20}$ P. Calabrese, J. Cardy, and E. Tonni, J. Phys. A 48, 015006 (2015).

21 S. Furukawa, V. Pasquier, and J. Shiraishi, Phys. Rev. Lett. 102, 170602 (2009); P. Calabrese, J. Cardy, and E. Tonni, J. Stat. Mech. P11001 (2009); P. Calabrese, J. Cardy, and E. Tonni, J. Stat. Mech. P01021 (2011); V. Alba, L. Tagliacozzo, and P. Calabrese, J. Stat. Mech. P06012 (2011); A. Coser, L. Tagliacozzo, and E. Tonni, J. Stat. Mech. (2014) P01008.

22 M. Kulaxizi, A. Parnachev, and G. Policastro, JHEP 1409 (2014) 010.
23 A. Coser, E. Tonni and P. Calabrese, J. Stat. Mech. P12017 (2014).

24 M. Hoogeveen and B. Doyon, Nucl. Phys. B 898, 78 (2015).

25 V. Eisler and Z. Zimboras, New J. Phys. 17053048 (2015).

${ }^{26}$ X. Wen, P.-Y. Chang, and S. Ryu, Phys. Rev. B 92, 075109 (2015).

27 M. Rangamani, and M. Rota, JHEP 1410 (2014) 060; E. Perlmutter, M. Rangamani, and M. Rota, Phys. Rev. Lett. 115, 171601 (2015).

28 O. Blondeau-Fournier, O. Castro-Alvaredo, and B. Doyon, arXiv:1508.04026.

29 M. Levin and X.-G. Wen, Phys. Rev. Lett. 96, 110405 (2006).

30 A. Y. Kitaev and J. Preskill, Phys. Rev. Lett. 96, 110404 (2006).

31 Y. A. Lee and G. Vidal, Phys. Rev. A 88, 042318 (2013).

32 C. Castelnovo, Phys. Rev. A 88, 042319 (2013); C. Castelnovo, Phys. Rev. A 89, 042333 (2014).

33 A. Bayat, S. Bose, P. Sodano, and H. Johannesson, Phys. Rev. Lett. 109, 066403 (2012); A. Bayat, P. Sodano, and S. Bose, Phys. Rev. B 81, 064429 (2010).

34 A. Bayat, H. Johannesson, S. Bose, and P. Sodano, Nat. Comm. 5, 3784 (2014).

35 B. Alkurtass, A. Bayat, I. Affleck, S. Bose, H. Johannesson, P. Sodano, E. S. Sorensen, and K. Le Hur, Phys. Rev. B 93, 081106 (2016).

36 A. Coser, E. Tonni, and P. Calabrese, J. Stat. Mech. (2015) P08005.

37 A. Coser, E. Tonni, and P. Calabrese, J. Stat. Mech. (2016) 033116.

38 A. Coser, E. Tonni, and P. Calabrese, arXiv:1511.08328.

39 P.-Y. Chang and X. Wen, arXiv:1601.07492.

40 C. P. Herzog and Y. Wang, arXiv:1601.00678.

${ }^{41}$ K. Audenaert, J. Eisert, M. B. Plenio, and R. F. Werner, Phys. Rev. A 66, 042327 (2002).

42 V. Eisler and Z. Zimboras, Phys. Rev. B 93, 115148 (2016).

43 C. De Nobili, A. Coser, and E. Tonni, arXiv:1604.02609.

44 X. Wen, S. Matsura, and S. Ryu, arXiv:1603.08534.

45 V. Alba, J. Stat. Mech. (2013) P05013.

46 C.-M. Chung, V. Alba, L. Bonnes, P. Chen, and A. M. Läuchli, Phys. Rev. B 90, 064401 (2014).

47 C. De Nobili, A. Coser, and E. Tonni, J. Stat. Mech. (2015) P06021

48 G. Refael and J. E. Moore, J. Phys. A 42, 504010 (2009). 
49 G. Refael and J. E. Moore, Phys. Rev. Lett. 93, 260602 (2004).

50 N. Laflorencie, Phys. Rev. B 72, 140408(R) (2005).

51 G. De Chiara, S. Montangero, P. Calabrese, and R. Fazio, J. Stat. Mech. (2006) P03001.

${ }^{52}$ M. Fagotti, P. Calabrese and J. E. Moore, Phys. Rev. B 83, 045110 (2011).

53 M. Pouranvari and K. Yang, Phys. Rev. B 88, 075123 (2013).

${ }^{54}$ G. Ramirez, J. Rodriguez-Laguna, and G. Sierra, J. Stat. Mech. (2014) P07003.

${ }_{55}$ R. Santachiara, J. Stat. Mech. (2006) L06002.

56 G. Refael and J. E. Moore, Phys. Rev. B 76, 024419 (2007).

57 J. A. Hoyos, A. P. Vieira, N. Laflorencie, and E. Miranda Phys. Rev. B 76, 174425 (2007).

58 Y.-C. Lin, F. Igloi, and H. Rieger, Phys. Rev. Lett. 99, 147202 (2007).

59 N. E. Bonesteel and K. Yang, Phys. Rev. Lett. 99, 140405 (2007).

60 D. Binosi, G. De Chiara, S. Montangero, and A. Recati Phys. Rev. B 76, 140405 (2007).

${ }^{61}$ F. Igloi and Y.-C. Lin, J. Stat. Mech. P06004 (2008).

${ }^{62}$ L. Fidkowski, G. Refael, N. E. Bonesteel, and J. E. Moore, Phys. Rev. B 78, 224204 (2008).

${ }^{63}$ R. Yu, H. Saleur, and S. Haas, Phys. Rev. B 77, 140402 (2008).

64 J. A. Hoyos, N. Laflorencie, A. P. Vieira, and T. Vojta, Europhys. Lett. 93, 30004 (2011).

65 I. A. Kovacs, and F. Igloi, Phys. Rev. B 80, 214416 (2009) I. A. Kovacs, and F. Igloi, EPL 97, 67009 (2012).

66 J. C. Getelina, F. C. Alcaraz, and J. A. Hoyos, Phys. Rev. B 93, 045136 (2016).

67 J. Rodrguez-Laguna, S. N. Santalla, G. Ramirez, and G. Sierra, 1601.03408.

${ }^{68}$ C. K. Burrell and T. J. Osborne, Phys. Rev. Lett. 99, 167201 (2007).

69 F. Igloi, Z. Szatmari, and Y.-C. Lin, Phys. Rev. B 85, 094417 (2012).

70 J. H. Bardarson, F. Pollmann, and J. E. Moore, Phys. Rev. Lett. 109, 017202 (2012).

71 M. Serbyn, Z. Papic, and D. A. Abanin, Phys. Rev. Lett. 110, 260601 (2013).
72 R. Vosk and E. Altman, Phys. Rev. Lett. 112, 217204 (2014); E. Altman and R. Vosk, Ann. Rev. Cond. Matt. Phys. 6, 383 (2015).

73 D. Pekker, G. Refael, E. Altman, E. Demler, and V. Oganesyan, Phys. Rev. X 4, 011052 (2014).

${ }^{74}$ Y. Zhao, F. Andraschko, and J. Sirker, arXiv:1602.04380.

75 R. Vasseur and J. E. Moore, Phys. Rev. B 92, 054203 (2015).

76 V. Alba, S. Inglis, and L. Pollet, Phys. Rev. B 93, 094404 (2016)

77 F. Igloi and C. Monthus, Phys. Rep. 412, 277 (2005).

78 Our DMRG code is implemented using the ITENSOR library, http://itensor.org/.

79 S. K. Ma, C. Dasgupta and C. K. Hu, Phys. Rev. Lett. 43, 1434 (1979); C. Dasgupta and S. K. Ma, Phys. Rev. B 22, 1305 (1980).

${ }^{80}$ F. Iglói, R. Juhász, and H. Rieger, Phys. Rev. B 61, 11552 (2000).

81 D. S. Fisher, Phys. Rev. B 50, 3799 (1994); D. S. Fisher, Phys. Rev. B 51, 6411 (1995).

82 R. Santos, V. Korepin, and S. Bose, Phys. Rev. A 84, 062307 (2011); R. Santos and V. Korepin, J. Phys. A 45, 125307 (2012). R. Santos and V. Korepin, arXiv:1603.05185.

83 P. Calabrese and J. Cardy, J. Stat. Mech. P06002 (2004).

84 P. Calabrese and J. Cardy, J. Phys. A 42, 504005 (2009).

85 P. Calabrese and A. Lefevre, Phys. Rev. A 78, 032329 (2008).

86 P. Calabrese, M. Mintchev, and E. Vicari, J. Stat. Mech. P09028 (2011).

87 P. Pippan, S. R. White, and H. G. Evertz, Phys. Rev. B 81, 081103(R) (2010).

88 I. Peschel, M. Kaulke, and O. Legeza, Ann. Physik (Lipzig) 8, 153 (1999).

89 I. Peschel and M.-C. Chung, J. Phys. A 32, 8419 (1999).

90 M.-C. Chung and I. Peschel, Phys. Rev. B 64, 064412 (2001).

91 I. Peschel, J. Phys. A 36, L205 (2003).

92 I. Peschel, J. Stat. Mech. (2004) P06004.

93 I. Peschel and V. Eisler, J. Phys. A 42, 504003 (2009). 